BU-991-M

\title{
Epidemiological Models with Age Structure, Proportionate Mixing, and Cross-Immunity
}

\author{
Carlos Castillo-Chavez ${ }^{1,2,5}$, Herbert W. Hethcote ${ }^{3}$, Viggo Andreasen ${ }^{1,2}$, \\ Simon A. Levin ${ }^{1,2,4}$, Wei-min Liu $^{1,2}$
}

\begin{abstract}
Infection by one strain of influenza type A provides some protection (cross-immunity) against infection by a related strain. It is important to determine how this influences the observed co-circulation of comparatively minor variants of the H1N1 and H3N2 subtypes. To this end, we formulate discrete and continuous time models with two viral strains, cross-immunity, age structure, and infectious disease dynamics. Simulation and analysis of models with cross-immunity indicate that sustained oscillations cannot be maintained by age-specific infection activity level rates when the mortality rate is constant; but are possible if mortalities are age-specific, even if activity levels are independent of age. Sustained oscillations do not seem possible for a single-strain model, even in the presence of age-specific mortalities; and thus it is suggested that the interplay between cross-immunity and age-specific mortalities may underlie observed oscillations.
\end{abstract}

\section{Introduction}

Infectious diseases like measles and influenza have several features in common; they cause recurrent epidemics and have strongly age-dependent contact rates. However, there are important differences. Measles is generated by a single infectious viral agent and hence individuals acquire permanent immunity after recovery. The situation with influenza is much more complex (see Palese and Young, 1982; Beveridge, 1977; Selby, 1976; Stuart-Harris and Schild, 1976; Dowdle et al., 1974; Fox and Kilbourne, 1973). There are three major recognized types of influenza: A (the most severe), B, and C; and each has various subtypes. For example, three recognized subtypes of type A - H1N1, H2N2, and $\mathrm{H} 3 \mathrm{~N} 2$ - have been isolated from man; and there are several recognized strains (comparatively

1. Ecology and Systematics, Corson Hall, Comell University, Ithaca, NY 14853

2. Center for Applied Mathematics, Cornell University

3. Department of Mathematics, University of Iowa, Iowa City, Iowa 52242

4. Ecosystem Research Center, Comell University

5. Biometrics Unit, Warren Hall, Comell University 
minor variants) within each subtype. The appearance of several strains is due to the capacity of influenza viruses to change their antigenic structure (see Webster, et al., 1982), which effectively allows them to circumvent individuals' immune responses. Unfortunately, the development of vaccines and vaccination programs, which have been successful for diseases such as smallpox, measles and poliomyelitis, is greatly complicated by an ever-changing virus.

In general, influenza (A) epidemics have severe effects on the human population and occasionally are responsible for pandemics, such as the infamous one of 1918 , which affected $30 \%$ to $60 \%$ of the population. These pandemics could result from the generation of novel subtypes, or they could result from high population susceptibility to a reappearing old subtype. In the latter case, as for the reappearance of the H1N1 subtype in 1977-78, the inter-epidemic time must depend on the time for a sufficient susceptible pool to develop.

Because of the complexity and severity of the disease, it is of utmost importance to determine the mechanisms responsible for the dynamics of influenza. The observed patterns associated with influenza include: secondary waves striking a community soon after a first attack, epidemics (occurring annually between pandemics and involving successive drift variants of previous pandemic subtypes), and worldwide pandemics occurring at approximately 10-to-40-year intervals (Fine, 1982). Central issues currently under exploration by various investigators include study of the extent that these patterns reflect the influence of antigenic variants, community structure, weather, and/or geography.

A specific question of interest to us deals with the possible mechanisms underlying the recurrence of epidemics and the persistence of co-circulating virus types between pandemics. In related work, Liu (1988) extends these approaches to consider the interaction of the human population with other host populations, which may be a source of recombinants or mutants

In Section 2, we introduce a two-strain discrete-time model with cross-immunity. This model incorporates age structure via a contact matrix (under the assumption of proportionate mixing) and agespecific mortality. This model has been used primarily as an exploratory simulation tool in our attempts to understand the dynamics generated by the highly heterogeneous interactions of this hostpathogen system. The results of extensive but not exhaustive simulations, also recorded in this section, 
show extrem. y complicated dynamics, ranging from damped oscillations and sustained periodic behavior to chaotic behavior, depending on the degree of cross-immunity between strains and on the age-step used. Because of the difficulty in analyzing this model, we turn in the following sections to continuous versions. and systematically incorporate some of the complexities of the discrete-time model. Differences remain, however, some of which relate to inherent properties of discrete-time models; therefore, some issues remain unresolved.

In Section 3, we extend the Dietz-Elveback continuous time model (see Dietz, 1979) for homogeneous populations to include two strains with different degrees of cross-immunity. Our mathematical analysis of this model indicates results coincident with the dynamic behavior observed in our simulation model with age structure removed. Sections 4 and 5 consider a one-strain continuous model with age structure. A threshold condition is computed, and a Lotka-like characteristic equation is obtained. We then proceed with a preliminary local stability analysis of the steady-state age distributions and establish (numerically) the impossibility of bifurcating periodic solutions for a two-step age-specific contact rate. After a continuous two strain age-dependent model with partial cross-immunity is introduced in Section 6 , we proceed to obtain partial local stability results for a particular case of the continuous two-strain age-dependent model. We then comment on the relevance of these results to epidemiological studies. Finally, in a series of appendices, we collect the mathematical details and show that the models introduced in Sections 4 and 6 are well-posed.

\section{The two-strain simulation model}

The model presented in this section subdivides the population into discrete age classes, and incorporate age-specific mortalities and contact rates, discrete time steps and age-dependent disease dynamics. To introduce the algorithm for the model, we need some notation. In what follows, the sub-index $i$ indicates that the corresponding class has been infected by or recovered from strain $i$. If two related strains of a virus such as influenza are co-circulating in a population, then individuals who have been infected by one strain may have partial immunity (i.e., decreased susceptibility) to the other strain (see Castillo-Chavez, et al., 1987; Couch and Kasel, 1983). Assume that individuals, while infected with one strain, temporarily are not susceptible to the other, either because of temporary immunity or 
because of isolation from the rest of the population. Assume further that an individual, once recovered from one strain of influenza, has permanent immunity to that strain but is susceptible to the other strain with, perhaps, a reduced level of susceptibility due to partial immunity.

In the transfer diagram (Fig. 2), $X$ is the susceptible class, $Y_{i}$ denotes those infected by strain $i$ but still susceptible to the other strain, and $Z_{i}$ denotes those recovered from strain $i$. We introduce $\sigma_{j}$ as the relative susceptibility to strain $j(j \neq i)$ for an individual that has been infected by $i$. Note that $\sigma=1$ corresponds to no cross-immunity, whereas $\sigma=0$ corresponds to total cross-immunity. $V_{i}$ denotes those infected with strain $i$ but recovered from the other strain, and $W$ denotes those recovered from both strains.

The population is divided into 80 one-year (360 day) age classes or compartments, with a fixed (steady-state) fraction $F_{k}$ of the population being in compartment $k$. Note that, as is standard in such models, we balance births and deaths to hold population size constant; since there is no disease induced mortality assumed, we also assume a stationary age distribution. In general, then, $F_{k+1}=P_{k} F_{k}$, where $P_{k}$ is the survival probability from year $k$ to year $k+1$. Let $X_{k}^{t}, Y_{1 k}^{t}, Y_{2 k}^{t}, Z_{1 k}^{t}, Z_{2 k}^{t}, V_{1 k}^{t}, V_{2 k}^{t}$ and $W_{k}^{t}$ be the fractions of the total population at time step $t$ in age compartment $k$ and the indicated epidemiological class. Note that

$$
X_{k}^{t}+Y_{1 k}^{t}+Y_{2 k}^{t}+Z_{1 k}^{t}+Z_{2 k}^{t}+V_{1 k}^{t}+V_{2 k}^{t}+W_{k}^{t}=F_{k}
$$

for all $t$ and all $k$. The daily activity level $a_{k}$ of age group $k$ is a measure of the relative amount of mixing done by age group $k$ when compared to other age groups. The proportionate mixing assumption specifies that the number of daily contacts of an infective in group $l$ is proportional to activity level $a_{l}$, and that these contacts are spread among the age groups in proportion to their activity levels $a_{k}$. Thus the daily contact rate of an infective in group $l$ with individuals in group $k$ is proportional to $a_{k} a_{l}$. The incidence into $Z_{i l}$ is similarly structured.

Motivated by the fact that the average infective period of influenza lies between 2 and 6 days, we have used a time step of 3 days. Our preliminary investigations of the effects of the time step, however, indicate that the choice of time step may be of crucial importance, especially as the time step is reduced to zero. It is clear that this requires further examination, especially to the extent that it affects 
the quantitative results. We introduce the infection at time step 0 by assuming that a fraction $\eta$ of the individuals in each compartment and class are infectious, and the remainder are susceptible. At time step $t$, the total infectivity of strain $i$ is calculated as $T_{i}=\sum_{l=1}^{80} a_{k}\left(Y_{i l}^{t}+V_{i l}^{t}\right), i=1,2$. Let $\beta_{i}$ be the incidence proportionality factor for a three-day period for strain $i$. This means that the incidences into the appropriate four age compartments $k$ due to the infectives of each strain at time step $t$ are as follows: the incidence into $Y_{1 k}^{t+1}$ is $\beta_{1} a_{k} X_{k}^{t} T_{1}$, the incidence into $Y_{2 k}^{t+1}$ is $\beta_{2} a_{k} X_{k}^{t} T_{2}$, the incidence into $V_{1 k}^{t+1}$ is $\sigma_{1} \beta_{1} a_{k} Z_{2}^{t} T_{1}$ and the incidence into $V_{2 k}^{t+1}$ is $\sigma_{2} \beta_{2} a_{k} Z_{1}^{t} T_{2}$. We make a simplification for computational purposes by ignoring age structure within each 30 -day month. All infectives recover after three days and move into the removed classes. After every 10 three-day time steps (one month), the fractions within each class in each subcompartment are adjusted to correspond to natural aging and mortality. Thus $\left(1-P_{k}\right) / 12$ of individuals in the susceptible, infectious and removed classes in age compartment $k$ are removed due to mortality, and the survivors are moved into the same epidemiological class in age compartment $k+1$. After all classes and compartments from age compartment 80 down to age compartment 1 have been adjusted, susceptible newborns are introduced; to account for these, we add $F_{1} / 12$ to $X_{1}^{t}$.

In order to approximate the age distribution in a developed country, we have chosen the fractions in the age compartments to be

$$
F_{k}= \begin{cases}1-0.2 k / 50, & 1 \leq k \leq 50 \\ 0.8-0.8(k-50) / 30, & 50 \leq k \leq 80\end{cases}
$$

so that $80 \%$ survive to age 50 and none survive past age 80 . One should, of course, explore other functional forms. The most important aspect of (2.1) is that it differs qualitatively from an exponential model, which has age-independent mortalities. The activity levels are chosen for five age categories: preschool (age 1 to 5), elementary school (age 6 to 12), secondary school (age 13 to 18), adults (age 19 to 60 ) and senior citizens (age 61 to 100 ). One would expect that the activity levels would be highest for the school children and lowest for the senior citizens. For these five groups, activity levels of 4:8:4:2:1 are consistent with the non-proportionate mixing contact matrices used by Longini et al. (1978) and Schenzle (1985). Other activity levels are also considered, as shown in Table 1. The 
incidence proportionality factors $\beta_{i}$ are chosen so that the calculated incidences are consistent with observed incidences for influenza. The 640 susceptible, infectious and removed class-compartments must be updated every time step; therefore, our analysis has been facilitated by implementation on the Cornell supercomputer (IBM 3090/4) with FPS (Floating Point System) array processors, because of its parallel processing capability. We emphasize that the numerical results for the two strain model to be discussed below are from the discrete time simulation model, and depend critically on the choice of time step.

The simulations indicate that for the symmetric contact case $\left(\sigma=\sigma_{1}=\sigma_{2}, \beta=\beta_{1}=\beta_{2}=0.2\right)$, if the two strains are strongly coupled, i.e., $\sigma$ is small, the system goes through cycles with a period of $10-20$ years, where each cycle may contain several outbreaks followed by a period with very low disease levels (Fig. 3). For intermediate coupling $(0.33 \leq \sigma \leq 0.8)$, we observe regular cycles with a period of about 40 months (Fig. 4) and with amplitude that increases with $\sigma$. For large $\sigma\left(\sigma_{1}=\sigma_{2} ; .8 \leq \sigma \leq 1.0\right)$, the amplitude decreases as the two strains become antigenically unrelated. In the transition between strong and intermediate coupling $(\sigma \approx 0.32)$, the system exhibits complicated dynamics, indicating a complex interaction between age-structure and cross-immunity (Fig. 5). When the two strains have equal cross immunity coefficients and different transmission rates $\beta_{1} \neq \beta_{2}$, we observe that the amplitude of the regular oscillation decreases for increasing $\left|\beta_{1}-\beta_{2}\right|$ (Fig. 6a, b). This indicates that as two strains become different, the diseases decouple and effectively act as two independent strains. In contrast, for equal transmission coefficients but different coefficients of cross-immunity $\sigma_{1} \neq \sigma_{2}$, the changes in the cycles are less pronounced (Fig. 7). The introduction of transmission rates that oscillate with small amplitudes due to natural seasonal fluctuations does not change qualitatively the observed periodic behavior for the appropriate parameter range (Fig. 8). The use of slightly fluctuating transmission rates can also be seen as the formal superposition of a forcing term to check the structural stability of the observed periodic solutions. For an extensive discussion of this model see Andreasen (1988a).

To understand better the mechanisms behind the observed sustained oscillations, we have performed further numerical experiments. When the age structure for the mechanism for transmission of the viral diseases is changed by setting all the activity levels equal to one, sustained oscillations are still 
observed for $\sigma=0.5$. If, however, instead of using the survivorship curve given by (2.1), we assume a constant death rate for the first 80 age classes followed by no survival into the 81 st age class, then the results change. This mortality structure resembles a negative exponential survivorship curve (see Hethcote, Stech \& Van den Driessche, 1981). In this situation, only a stable fixed point is observed. Finally, a set of numerical experiments have been performed in which a strain is removed from circulation by setting its corresponding transmission coefficient $\left(\beta_{i}\right)$ equal to zero. Using again the survivorship curve given by (2.1), we have performed extensive simulations (see Table 1) for different sets of activity levels. In all of these cases damped oscillations are observed. The dominant eigenvalues are complex, and their imaginary part $b$ may be estimated by $b=2 \pi / t_{r}$, where $t_{r}$ is the time for one revolution of the spiral. Then if $a$ is the real part of the complex eigenvalue and peaks of $\sum Y_{k}^{t}$ occur at times $t_{1}$ and $t_{2}, a$ can be estimated from

$$
e^{a\left(t_{2}-t_{1}\right)}=\frac{e^{a t_{2}}}{e^{a t_{1}}}=\frac{\sum Y_{k}^{t_{2}}-\sum Y_{k}^{e}}{\sum Y_{k}^{t_{1}}-\sum Y_{k}^{e}} .
$$

The large ratios of $b$ to $a$ in Table 1 indicate that the solutions spiral slowly into the equilibrium, so that they would be difficult to distinguish from periodic solutions if one observed them for a short time period. The equilibria in Table 1 are reached before 2000 years. These results are not surprising since it is generally believed that symmetric age-specific contact rates by themselves can at most drive slowly damped oscillations (for example, see Anderson and May, 1984; Schenzle, 1985). Our results suggest that age structure is important, but perhaps primarily in its direct effect on the fraction of susceptibles; that is, an age structure relatively skewed towards earlier age classes will have a higher proportion susceptible. We remark again that these simulations have provided us only with a suggestive picture that we feel deserves further investigation. To examine the results just outlined, in the following sections we look at a sequence of continuous time approximations which can be derived from the same basic principles used in the derivation of our simulation model.

\section{The continuous model for two strains without age structure}

- The model in this section incorporates the effects of two co-circulating strains into the dynamics of a homogeneous population. Let $X(t), Y(t), Z_{i}(t), V_{i}(t)$, and $W(t)$ denote the fractions in the 
respective classes as specified in Section 2 . Here, $\beta_{i}$ denotes the transmission coefficient of strain $i, \gamma_{i}$ denotes the recovery rate from strain $i, \mu$ denotes the constant mortality rate, and again, $\sigma_{j}$ represents the relative susceptibility of types $Z_{i}$ in terms of their acquisition of strain $j$ (i.e., the degree of crossimmunity). If we now follow the transfer diagram in Fig. 2, we arrive at the following set of equations:

$$
\begin{gathered}
X^{\prime}(t)=-\left[\beta_{1}\left(Y_{1}+V_{1}\right)+\beta_{2}\left(Y_{2}+V_{2}\right)\right] X+\mu-\mu X \\
Y_{i}^{\prime}(t)=\beta_{i}\left(Y_{i}+V_{i}\right) X-\left(\gamma_{i}+\mu\right) Y_{i} \\
Z_{i}^{\prime}(t)=\gamma_{i} Y_{i}-\left(\sigma_{j} \beta_{j}\left(Y_{j}+V_{j}\right)+\mu\right) Z_{i} \\
V_{i}^{\prime}(t)=\sigma_{i} \beta_{i}\left(Y_{i}+V_{i}\right) Z_{j}-\left(\gamma_{i}+\mu\right) V_{i} \\
W^{\prime}(t)=\gamma_{1} V_{1}+\gamma_{2} V_{2}-\mu W \\
X(0)=X_{0}, \quad Y_{i}(0)=Y_{i 0}, \quad Z_{i}(0)=Z_{i 0}, \quad V_{i}(0)=V_{i 0}, W(0)=W_{0}
\end{gathered}
$$

where $j=2$ if $i=1$ and $j=1$ if $i=2$. The equation (3.5) is redundant since for all times we have

$$
X(t)+Y_{1}(t)+Y_{2}(t)+Z_{1}(t)+Z_{2}(t)+V_{1}(t)+V_{2}(t)+W(t)=1
$$

The nonnegative orthant in $\mathbf{R}^{7}$ is positively invariant for (3.1)-(3.7) and unique solutions exist for all time. The contact number for strain $i$ is

$$
R_{i}=\frac{\beta_{i}}{\gamma_{i}+\mu}
$$

This model is similar to the model in Dietz (1979) except that his model has $\sigma_{1}=\sigma_{2}=1$ so that recovery from one strain does not reduce a person's susceptibility to the other strain. Dietz's model is based on the Monte Carlo simulation models of Elveback et al. (1964). Some of the analysis of the model above is similar to the analysis in Dietz (1979), so we omit some details.

The model has four equilibria $G_{i}$ for $i=1,2,3,4$. The trivial equilibrium $G_{1}$ has $X=1$ and all other variables equal to zero so that neither viral strain is present. If the contact numbers satisfy $R_{1} \leq 1$ and $R_{2} \leq 1$, then $G_{1}$ is the only equilibrium in the nonnegative orthant and all solutions in the nonnegative orthant approach $G_{1}$. The global asymptotic stability of $G_{1}$ in this case is shown by using the Lyapunov function $Y_{1}+V_{1}+Y_{2}+V_{2}$ and the Lyapunov-LaSalle theorem (Hale, 1969).

If $R_{1}>1$, then there is a boundary equilibrium $G_{2}$ given by

$$
G_{2}:\left(X, Y_{1}, Z_{1}, Y_{2}, Z_{2}, V_{1}, V_{2}\right)=\left(\frac{1}{R_{1}}, \frac{\mu}{\gamma_{1}+\mu}\left(1-\frac{1}{R_{1}}\right), \frac{\gamma_{1}}{\gamma_{1}+\mu}\left(1-\frac{1}{R_{1}}\right), 0,0,0,0\right)
$$

Analysis of the Jacobian of the system at $G_{2}$ reveals that $G_{2}$ is locally asymptotically stable if $R_{1}>1$ 
and

$$
R_{2}<\frac{R_{1}}{1+\sigma_{2}\left(R_{1}-1\right) \gamma_{1} /\left(\gamma_{1}+\mu\right)} .
$$

The equilibrium $G_{2}$ is an unstable saddle if condition (3.10) is not satisfied. If $R_{2}>1$, then there is an analogous boundary equilibrium $G_{3}$.

If the two conditions

$$
\begin{aligned}
& R_{2}>\frac{R_{1}}{1+\sigma_{2}\left(R_{1}-1\right) \gamma_{1} /\left(\gamma_{1}+\mu\right)} \\
& R_{1}>\frac{R_{2}}{1+\sigma_{1}\left(R_{2}-1\right) \gamma_{2} /\left(\gamma_{2}+\mu\right)}
\end{aligned}
$$

are both satisfied, then there is a nontrivial equilibrium $G_{4}$ at which both strains remain endemic. Equivalently, these may be written

$$
\frac{R_{1}}{1+\left(R_{1}-1\right) A}>R_{2}>\frac{R_{1}}{1+\left(R_{1}-1\right) B},
$$

where $B=\sigma_{2} \gamma_{1} /\left(\gamma_{1}+\mu\right)$ and $A=\left(1-\left(\sigma_{1} \gamma_{2} /\left(\gamma_{2}+\mu\right)\right)^{-1}\right)^{-1}$. Note that a necessary condition for this to occur is

$$
\frac{\gamma_{1}+\mu}{\gamma_{1} \sigma_{2}}+\frac{\gamma_{2}+\mu}{\gamma_{2} \sigma_{1}}<1
$$

If $\sigma_{1}=0$, then recovery from strain 2 also gives complete immunity to strain 1 so that no one ever enters class $V_{1}$. In this case threshold condition (3.12) reduces to $R_{1}>R_{2}$. If both $\sigma_{1}=0$ and $\sigma_{2}=0$, then states $V_{1}, V_{2}$ and $W$ are always empty since no one enters them. In this case the equilibrium $G_{2}$ is locally asymptotically stable if $R_{1}>1$ and $R_{1}>R_{2}$, while the equilibrium $G_{3}$ is locally asymptotically stable if $R_{2}>1$ and $R_{2}>R_{1}$. If $R_{1}=R_{2}>1$, then there is a line segment of neutrally stable equilibria joining $G_{2}$ and $G_{3}$, and which equilibrium is approached depends on the initial conditions. In the following, we assume that $\sigma_{1}>0$ or $\sigma_{2}>0$ to avoid the unusual case above.

If we let $\lambda_{1}$ and $\lambda_{2}$ be the $G_{4}$ equilibrium values of $\beta_{1}\left(Y_{1}+V_{1}\right)$ and $\beta_{2}\left(Y_{2}+V_{2}\right)$, respectively, then the coordinates of the equilibrium $G_{4}$ are

$$
\begin{gathered}
X=\frac{\mu}{\lambda_{1}+\lambda_{2}+\mu} \\
Y_{i}=\frac{\mu \lambda_{i}}{\left(\lambda_{1}+\lambda_{2}+\mu\right)\left(\gamma_{i}+\mu\right)} \\
Z_{i}=\frac{\mu \lambda_{i} \gamma_{i}}{\left(\lambda_{1}+\lambda_{2}+\mu\right)\left(\gamma_{i}+\mu\right)\left(\sigma_{j} \lambda_{j}+\mu\right)}
\end{gathered}
$$




$$
V_{i}=\frac{\mu \lambda_{j} \gamma_{j} \sigma_{i} \lambda_{i}}{\left(\lambda_{1}+\lambda_{2}+\mu\right)\left(\gamma_{j}+\mu\right)\left(\sigma_{i} \lambda_{i}+\mu\right)\left(\gamma_{i}+\mu\right)}
$$

where $j=2$ if $i=1$ and $j=1$ if $i=2$. The local stability of the equilibrium $G_{4}$ is not easy to analyze since one must show that all eigenvalues of the $7 \times 7$ Jacobian matrix at $G_{4}$ have negative real parts. However, numerical calculations with system (3.1)-(3.4) suggest that if (3.11) and (3.12) are satisfied and both $Y_{1}$ and $Y_{2}$ are initially positive, then all solutions in the nonnegative orthant approach the equilibrium $G_{4}$. The eigenvalues were computed for different values of $\sigma_{1}$ and $\sigma_{2}$. In all cases, we found that the eigenvalues have negative real parts. We note that the eigenvalue with largest real part usually has its real part around $-10^{-4}$ and its imaginary part around $10^{-2}$, so the imaginary part is approximately 100 times the real part. Hence solutions will spiral rapidly as they approach equilibrium. More detail on these simulations is shown in Table 2 .

When the viral strains are similar so that $\beta_{1}=\beta_{2}, \gamma_{1}=\gamma_{2}$, and $\sigma_{1}=\sigma_{2}$ and the initial conditions are symmetric, then $Y_{1}(t)=Y_{2}(t), Z_{1}(t)=Z_{2}(t)$ and $V_{1}(t)=V_{2}(t)$ for all time. In this case the system (3.1)(3.4) reduces to a four dimensional system. Furthermore, when the threshold condition $R=\beta /(\gamma+\mu)>1$ is satisfied, then there is a nontrivial equilibrium corresponding to the equilibrium $G_{4}$ for (3.1) to (3.4). This nontrivial equilibrium is locally asymptotically stable. This suggests that periodic solutions do not arise by Hopf bifurcation at the equilibrium point $G_{4}$ for the system (3.1)-(3.4). For further details we refer the reader to Appendix A.

\section{The threshold condition for the continuous age-structured one-strain model}

The basic epidemiological model for one strain of influenza virus (or one infectious agent) is formulated in terms of continuous variables. Then a threshold condition is obtained which determines the asymptotic steady-state age distributions. The population is separated into susceptible, infected, and removed classes, where $x(a, t), y(a, t)$ and $z(a, t)$ are the probability densities in these respective classes. In this SIR epidemiological model, $\int_{a_{1}}^{a_{2}} x(a, t) d a, \int_{a_{1}}^{a_{2}} y(a, t) d a, \int_{a_{1}}^{a_{2}} z(a, t) d a$, denote the proportions of the population in each class that have ages in the age-interval $\left(a_{1}, a_{2}\right)$ at time $t$. The transfer diagram for this model is that of Fig. 1. 
Assume that the population has reached a steady-state age distribution, that all newborns are susceptible, and that the transfer of infection is due to a proportionately-mixed age-dependent bilinear incidence rate. The dynamics of the classes are governed by the following initial boundary value problem (see Hoppensteadt, 1974b; Dietz, 1975; May, 1986; Schenzle, 1984; Dietz and Schenzle, 1985; Webb, 1985):

$$
\begin{gathered}
\frac{\partial x(a, t)}{\partial a}+\frac{\partial x(a, t)}{\partial t}=-\lambda(t) b(a) x(a, t)-\mu(a) x(a, t), \\
\frac{\partial y(a, t)}{\partial a}+\frac{\partial y(a, t)}{\partial t}=\lambda(t) b(a) x(a, t)-(\gamma+\mu(a)) y(a, t), \\
\frac{\partial z(a, t)}{\partial a}+\frac{\partial z(a, t)}{\partial t}=\gamma y(a, t)-\mu(a) z(a, t), \\
\lambda(t)=\beta \int_{0}^{\infty} b\left(a^{\prime}\right) y\left(a^{\prime}, t\right) d a^{\prime}, \\
x(a, 0)=x_{0}(a), \quad y(a, 0)=y_{0}(a), z(a, 0)=z_{0}(a), \\
x(0, t)=\rho=\frac{1}{\int_{0}^{\infty} e^{-M\left(a^{\prime}\right)} d a^{\prime}}, M(a)=\int_{0}^{a} \mu(\alpha) d \alpha, \quad y(0, t)=0=z(0, t) .
\end{gathered}
$$

where $\mu(a)$ is the age-specific mortality rate, $\rho$ is the birth rate, $\gamma$ is the constant recovery rate, $b(a)$ is the age-specific activity level, $\lambda(t)$ is the instantaneous force of infection, and $\beta$ is a transmission scaling factor. In this model the activity level between a susceptible person of age $a$ and an infected one of age $a^{\prime}$ is proportional to $b(a) b\left(a^{\prime}\right)$. This is a particular case of the so-called proportionate mixing assumption used by Barbour (1978), Nold (1980), Hethcote and Yorke (1984), Dietz and Schenzle (1985), and Hethcote and Van Ark (1987). The initial age distributions are assumed to be zero beyond some maximum age.

When the activity level $b(a)$ and the mortality rate $\mu(a)$ are independent of age $a$, then integration of the differential equations (4.1)-(4.6) over all ages leads to a time-dependent SIR model with vital dynamics. For this ordinary-differential-equation model involving the fraction of individuals in each class at time $t$, the contact number $R$ (basic reproduction number) is $\beta b^{2} /(\gamma+\mu)$. If $R \leq 1$, then the disease dies out; if $R>1$, then the fractions in each class approach endemic equilibrium values (Hethcote, 1976).

The model (4.1)-(4.6) is well-posed. The proof is found implicitly in Appendix D since this 
model is a special case of the two strain model that is shown to be well-posed there. In the remainder of this section we determine a threshold condition, that is, a quantity that must exceed one for the disease to remain endemic (persistent). This threshold condition was obtained previously by Dietz and Schenzle (1985) and also for a simpler model by Webb (1985). Due to its importance in our stability analysis and numerical experiments, a brief derivation of the threshold condition is presented.

Assume that the steady-state age distributions are reached as time approaches infinity, so that the force of infection asymptotically is a constant denoted by $\lambda^{*}$. The method of characteristics is used to obtain the following expressions for the steady-state age distributions.

$$
\begin{gathered}
x^{*}(a)=\rho e^{-\left[\lambda^{*} B(a)+M(a)\right]}, \\
y^{*}(a)=\rho e^{-M(a)} \int_{0}^{a} \lambda^{*} b\left(a^{\prime}\right) e^{-\lambda^{*} B\left(a^{\prime}\right)-\gamma\left(a-d^{\prime}\right)} d a^{\prime}, \\
z^{*}(a)=\rho e^{-M(a)}-x^{*}(a)-y^{*}(a),
\end{gathered}
$$

where

$$
B(a)=\int_{0}^{a} b(\alpha) d \alpha
$$

If we now substitute $y^{*}(a)$ into equation (4.4), we obtain that either $\lambda^{*}=0$ or else $\lambda^{*}$ satisfies the characteristic equation

$$
1=\beta \int_{0}^{\infty} b(a) \rho e^{-M(a)}\left(\int_{0}^{a} b(\alpha) e^{-\lambda^{*} B(\alpha)-\gamma(a-\alpha)} d \alpha\right) d a,
$$

which has a positive solution $\lambda^{*}$ provided that the threshold condition

$$
1<\beta \int_{0}^{\infty} b(a) \rho e^{-M(a)}\left(\int_{0}^{a} b(\alpha) e^{-\gamma(a-\alpha)} d \alpha\right) d a
$$

is satisfied.

Above the threshold (that is when inequality (4.12) is satisfied) the force of infection $\lambda^{*}$ is a positive constant, and equations (4.7)-(4.9) correspond to an endemic (persistent) steady-state age distribution. Below the threshold (that is when equation (4.12) is not satisfied), the force of infection $\lambda^{*}$ is zero and equations (4.7)-(4.9) correspond to a trivial steady-state age distribution where the disease has died out and there are no infected or recovered individuals. 


\section{Stability of the steady-state age distributions for the continuous one-strain model}

We now proceed with a local stability analysis of the steady-state age distributions of the model of Section 4 by taking perturbations of the steady states:

$$
\begin{gathered}
x(a, t)=x^{*}(a)+\xi(a, t), \\
y(a, t)=y^{*}(a)+\eta(a, t), \\
\lambda(t)=\lambda^{*}+\theta(t) .
\end{gathered}
$$

A linearization approach leads to the following first order approximate model for $\xi$, $\eta$, and $\theta$ :

$$
\begin{gathered}
\frac{\partial \xi}{\partial a}+\frac{\partial \xi}{\partial t}=-\lambda^{*} b(a) \xi-\theta(t) b(a) x^{*}(a)-\mu(a) \xi \\
\frac{\partial \eta}{\partial a}+\frac{\partial \eta}{\partial t}=\lambda^{*} b(a) \xi+\theta(t) b(a) x^{*}(a)-(\gamma+\mu(a)) \eta \\
\theta(t)=\beta \int_{0}^{\infty} b(a) \eta(a, t) d a, \\
\xi(0, t)=\eta(0, t)=0, \\
\xi(a, 0)=x_{0}(a)-x^{*}(a), \eta(a, 0)=y_{0}(a)-y^{*}(a) .
\end{gathered}
$$

If there exists a solution of (5.4)-(5.8) in separable form $\xi(a, t)=\xi(a) f(t)$, then it corresponds to a steady-state age distribution since the fractions in any age bracket are constant. Since the right hand sides of (5.4)-(5.6) are linear in the parts of the separable forms involving $t$, these parts involve an exponential in $t$. Thus we restrict ourselves to perturbations of the form

$$
\begin{gathered}
\xi(a, t)=\hat{\xi}(a) e^{p t}, \\
\eta(a, t)=\hat{\eta}(a) e^{p t}, \\
\theta(t)=\hat{\theta} e^{p t}, \hat{\theta} \text { a constant. }
\end{gathered}
$$

A straightforward computation shows that

$$
\begin{gathered}
\xi(a)=-\rho \hat{\theta} e^{-\left[\lambda^{*} B(a)+M(a)\right]} \int_{0}^{a} b(\alpha) e^{-p(a-\alpha)} d \alpha, \\
\eta(a)=-\hat{\theta} \rho e^{-M(a)} \int_{0}^{a} b\left(a^{\prime}\right) e^{-\left[(p+\gamma)\left(a-a^{\prime}\right)+\lambda^{*} B\left(a^{\prime}\right)\right]}\left[1-\lambda^{*} \int_{0}^{a^{\prime}} b(\alpha) e^{-p\left(\alpha^{\prime}-\alpha\right)} d \alpha\right] d a^{\prime}, \\
\hat{\theta}=\beta \int_{0}^{\infty} b(a) \eta(a) d a .
\end{gathered}
$$

Putting equation (5.13) into equation (5.14) leads (provided that $\hat{\theta} \neq 0$ ) to a Lotka-type characteristic equation for $p$ :

$$
1=\beta \int_{0}^{\infty} b(a) \rho e^{-M(a)}\left\{\int_{0}^{a} b\left(a^{\prime}\right) e^{-\gamma\left(a-a^{\prime}\right)-\lambda^{*} B\left(a^{\prime}\right)}\left[e^{-p\left(a-\alpha^{\prime}\right)}-\lambda^{*} \int_{0}^{a^{\prime}} b(\alpha) e^{-p(a-\alpha)} d \alpha\right] d a^{\prime}\right\} d a .
$$


If all roots of (5.15) have negative real parts, then all solutions of the form (5.9)-(5.11) will tend to zero as $t$ tends to infinity. Using the threshold condition given by the expression in (4.12) so that $\lambda^{*}>^{\wedge} 0$, we quickly find that no non-negative $p$ can satisfy equation (5.15). The study of the nature of all the roots of equation (5.15) is more difficult. Nevertheless, in some particular instances we have determined numerically that their corresponding nontrivial endemic steady-state age distributions are locally asymptotically stable.

For the trivial steady-state age distribution $\left(\lambda^{*}=0\right)$, equation (5.15) becomes:

$$
1=\beta \int_{0}^{\infty} b(a) \rho e^{-M(a)}\left[\int_{0}^{a} b\left(a^{\prime}\right) e^{-\gamma\left(a-a^{\prime}\right)-p\left(a-a^{\prime}\right)} d a^{\prime}\right] d a .
$$

If equation (4.12) is not satisfied, then the monotone character of the integrand in equation (5.16) implies that it has a unique real root $p_{0} \leq 0$, and that $p_{0}=0$ only at the threshold. It is also clear that complex roots (if any) appear in conjugate pairs. If we now let $p=r+i s$ denote a complex root in (5.16) with $s>0$, then by equating real and imaginary parts we observe that

$$
1=\beta \int_{0}^{\infty} b(a) \rho e^{-M(a)} \int_{0}^{a} b(a-\theta) e^{-(\gamma+r) \theta} \cos (s \theta) d \theta d a
$$

and since $\cos (s \theta)<1$ for some values of $\theta$ in the range of integration, it follows that

$$
\beta \int_{0}^{\infty} b(a) \rho e^{-M(a)} \int_{0}^{a} b(a-\theta) e^{-(\gamma+r) \theta} d \theta d a>1
$$

From this we conclude using (5.16) that $r<p_{0} \leq 0$. Hence if we are strictly below the threshold, then the trivial steady-state age distribution is locally asymptotically stable, at least for perturbations of the form (5.9)-(5.11). If inequality (4.12) is satisfied, then (5.16) has a unique positive root $p_{0}$ so that the trivial steady-state age distribution is unstable. In this case the disease does not die out, but persists.

In the remainder of this section we indicate how a numerical analysis of a particular case for equation (5.15) leads us to believe that the one-strain model without age-specific mortality rate $\mu(a)=\mu$ (a constant) has an endemic equilibrium point that is locally exponentially stable. In this particular case we take the age-specific activity level rate to be

$$
b(a)= \begin{cases}1, & 0 \leq a<C, \\ D, & C \leq a<\infty, 0<D \neq 1 .\end{cases}
$$


In this case the threshold quantity in equation (4.12) becomes

$$
\beta \mu\left[\frac{1}{\mu(\mu+\gamma)}+\frac{(1-D) e^{-(\mu+\gamma) C}}{(\mu+\gamma) \gamma}+e^{-\mu C}\left(\frac{D^{2}}{\mu(\mu+\gamma)}+\frac{D}{\gamma(\mu+\gamma)}-\frac{1}{\eta \mu}\right)\right],
$$

the characteristic equation is given by

$$
\begin{gathered}
\frac{1}{\left(\mu+\lambda^{*}\right)(\mu+\gamma)}+\frac{(1-D) e^{-(\mu+\gamma) c}}{(\mu+\gamma)\left(\gamma-\lambda^{*}\right)} \\
+e^{-\left(\mu+\lambda^{*}\right) c}\left[\frac{D^{2}}{\left(\mu+\lambda^{*} D\right)(\mu+\gamma)}+\frac{D}{\left(\gamma-\lambda^{*}\right)(\mu+\gamma)}-\frac{1}{\left(\gamma-\lambda^{*}\right)\left(\mu+\lambda^{*}\right)}\right]-\frac{1}{\mu \beta}=0,
\end{gathered}
$$

and equation (5.15) reduces to

$$
\begin{gathered}
\frac{\left(1-\frac{\lambda^{*}}{p}\right)\left[1-e^{-\left(\mu+\lambda^{*}\right) c}\right]}{\left(\mu+\lambda^{*}\right)\left(\gamma-\lambda^{*}+p\right)}+\frac{\frac{\lambda^{*}}{p}\left[1-e^{-\left(\mu+\lambda^{*}+p\right) c}\right]}{\left(\gamma-\lambda^{*}\right)\left(\mu+\lambda^{*}+p\right)}-\frac{\gamma\left[1+(D-1) e^{-(\mu+\gamma+p)}\right]}{\left(\gamma-\lambda^{*}+p\right)\left(\gamma-\lambda^{*}\right)(\mu+\gamma+p)} \\
+\frac{e^{-\left(\mu+\lambda^{*}\right) c} D}{\mu+\gamma+p}\left[\frac{1-\frac{\lambda^{*}}{p}}{\gamma-\lambda^{*}+p}+\frac{D(\mu+p)}{\left(\mu+\lambda^{*} D\right)\left(\mu+\lambda^{*} D+p\right)}-\frac{D \frac{\lambda^{*}}{p}}{\mu+\lambda^{*} D+p}\right] \\
+\frac{D \frac{\lambda^{*}}{p} e^{-\left(\mu+\lambda^{*}+p\right) c}}{\mu+\gamma+p}\left[\frac{1}{\gamma-\lambda^{*}}+\frac{D}{\mu+\lambda^{*} D+p}\right]-\frac{1}{\beta \mu}=0 .
\end{gathered}
$$

The numerical procedure computes the threshold quantity from equation (5.20). If it is greater than one, then the characteristic equation (5.21) is solved for $\lambda^{*}$. Using the force of infection $\lambda^{*}$, we solved the equation (5.22) numerically for $p$. For example, if the average infectious period $1 / \gamma$ is 1 week, the average lifetime $1 / \mu$ is 70 years, $C=5$ years, $D=4$ and $\beta=0.2$, then the threshold quantity (5.20) is 2.99 , the solution of the characteristic equation $(5.21)$ is $\lambda^{*}=.000132$ and the roots of (5.22) with largest real part are $p=-0.000693 \pm i 0.0228$. Results of numerous runs with reasonable epidemiological parameters for influenza, different values of $D$ and $C$, suggest strongly that the $p$ roots always have negative real parts so that (at least for these particular cases) the stable age distribution is locally asymptotically stable for the one-strain continuous model. We note that for this $b(a)$, the $p$ root with the largest real part usually has its real part around $-10^{-3}$ and its imaginary part around $10^{-2}$ so the imaginary part is approximately 10 times the real part. Since the solutions are very weakly damped, they would oscillate rapidly as they approach the equilibrium solution and might look numerically like periodic solutions.

Calculations with the one-strain model in Section 2 are consistent with the results above. Thus age-specific activity levels do not seem to lead to periodic solutions in the one-strain model. Further- 
more, simulations with different age-specific mortalities do not lead to periodic solutions.

Other investigators have also shown that models with age-specific mortality do not lead to periodic solutions. Andreasen (1988a, 1988b) considers the model (4.1)-(4.6) with constant ageindependent activity level $(b(a)=1)$ and age-specific mortality corresponding to a fixed lifespan of $\mathrm{A}$ years for every individual. He approximates the Lotka-type characteristic equation (5.15) by observing that the infectious period is four orders of magnitude smaller than the lifespan A of the host. The approximate $\mathrm{p}$ roots suggest that solutions are slowly damped with the period given by $2 \pi \sqrt{K D}$ where $D=1 / \gamma$ is the average infectious period, and $K$ is the average age at infection.

\section{The model for two strains with partial cross-immunity}

If two related strains of a virus such as influenza both are circulating in a population, then individuals who have been infected by one strain may have partial immunity (i.e., decreased susceptibility) to the other strain. The two-strain model here is the continuous analog of the simulation model in Section 2. In what follows, the sub-index $i$ indicates that its class is infected or recovered from influenza due to strain $i$. Assume, as before, that those individuals infected with one strain are not susceptible to the other, and that an individual once recovered from a strain of influenza, has permanent immunity to this strain, and possibly a reduced level of susceptibility to the other strain.

The initial boundary value problem governing the dynamics of these classes under proportionately mixed age-dependent bilinear incidence rates and the assumptions of sections 2 and 4 is:

$$
\begin{gathered}
\frac{\partial x}{\partial a}+\frac{\partial x}{\partial t}=-\lambda_{1}(t) b(a) x(a, t)-\lambda_{2}(t) b(a) x(a, t)-\mu(a) x(a, t), \\
\frac{\partial y_{i}}{\partial a}+\frac{\partial y_{i}}{\partial t}=\lambda_{i}(t) b(a) x(a, t)-\gamma_{i} y_{i}(a, t)-\mu(a) y_{i}(a, t), \\
\frac{\partial z_{i}}{\partial a}+\frac{\partial z_{i}}{\partial t}=\gamma_{i} y_{i}(a, t)-\sigma_{j} \lambda_{j}(t) b(a) z_{i}(a, t)-\mu(a) z_{i}(a, t), \\
\frac{\partial v_{i}}{\partial a}+\frac{\partial v_{i}}{\partial t}=\sigma_{i} \lambda_{i}(t) b(a) z_{j}(a, t)-\gamma_{i} v_{i}(a, t)-\mu(a) v_{i}(a, t), \\
\frac{\partial w}{\partial a}+\frac{\partial w}{\partial t}=\gamma_{1} v_{1}(a, t)+\gamma_{2} v_{2}(a, t)-\mu(a) w(a, t), \\
x(0, t)=\rho=\frac{1}{\lambda_{i}(t)=\beta_{i} \int_{0}^{\infty} b\left(a^{\prime}\right)\left[y_{i}\left(a^{\prime}, t\right)+v_{i}\left(a^{\prime}, t\right)\right] d a^{\prime},} \\
\int_{0}^{\infty} e^{-M\left(a^{\prime}\right)} d a^{\prime}
\end{gathered}
$$




$$
\begin{gathered}
x(a, 0)=x_{0}(a), y_{i}(a, 0)=y_{0 i}(a), v_{i}(a, 0)=v_{0 i}(a), \\
z_{i}(a, 0)=z_{0 i}(a), w(a, 0)=w_{0}(a),
\end{gathered}
$$

where $i=1,2, j=1$ if $i=2, j=2$ if $i=1, \gamma_{i}$ denotes constant recovery rate, and $\mu(a)$ and $b(a)$ denote the age-specific mortality and activity level rates respectively. The transmission scaling factors here are $\beta_{1}$ and $\beta_{2}$. The susceptibility factors $\sigma_{1}$ and $\sigma_{2}$, which are between 0 and 1 , are measures of the crossimmunity. Note that the one strain model in section 4 is a special case with $\beta_{2}=0$. If the age specific activity level $b(a)$ and the mortality rate $\mu(a)$ are constant, then the integration over all ages of the differential equations in the model above leads to the two-strain model in Section 3.

The well-posedness of this two-strain model is established in Appendix D. If we now assume that the steady-state age distributions are approached as time approaches infinity, so that the forces of infection asymptotically are constants denoted by $\lambda_{1}^{*}$ and $\lambda_{2}^{*}$, then proceeding as in section 4 , we obtain that either $\lambda_{i}^{*}=0$ or $\lambda_{i}^{*}$ satisfies the characteristic equations

$$
\begin{gathered}
1=\beta_{i} \int_{0}^{\infty} b(a) \rho e^{-M(a)}\left[\int _ { 0 } ^ { a } b ( \alpha ) e ^ { - \gamma _ { i } ( a - \alpha ) } \left(e^{-\left(\lambda_{1}^{*}+\lambda_{i}^{*}\right) B(\alpha)}\right.\right. \\
\left.\left.+\sigma_{i} \gamma_{j} \lambda_{j}^{*} \int_{0}^{\alpha} e^{-\sigma_{i} \lambda_{i}^{*}(B(\alpha)-B(\theta))}\left[\int_{0}^{\theta} b(\phi) e^{-\gamma_{j}(\theta-\phi)-\left(\lambda_{1}^{*}+\lambda_{2}^{*}\right) B(\phi)} d \phi\right] d \theta\right) d \alpha\right] d a,
\end{gathered}
$$

where $M(a)=\int_{0}^{a} \mu(\alpha) d \alpha$ and $B(a)=\int_{0}^{a} b(\alpha) d \alpha$. The two characteristic equations correspond to $(i, j)=(1,2)$ and $(i, j)=(2,1)$. We have not found threshold conditions that are necessary and sufficient conditions for the two characteristic equations to have positive solutions $\lambda_{1}^{*}$ and $\lambda_{2}^{*}$ (for further details see Appendix B).

We now proceed to linearize the system (6.1)-(6.8) around its steady-state age distributions as in Section 5 (details are found in Appendix C). If we assume that

$$
\begin{gathered}
x(a, t)=x^{*}(a)+\hat{\xi}(a) e^{p t}, \\
y_{i}(a, t)=y_{i}^{*}(a)+\hat{\eta}_{i}(a) e^{p t}, \\
z_{i}(a, t)=z_{i}^{*}(a)+\hat{\rho}_{i}(a) e^{p t}, \\
v_{i}(a, t)=v_{i}^{*}(a)+\hat{\omega}_{i}(a) e^{p t}, \\
\lambda_{i}(t)=\lambda_{i}^{*}+\hat{\theta}_{i} e^{p t},
\end{gathered}
$$

for $i=1,2$, then we arrive at the following Lotka-type characteristic equations for $p$ : 


$$
\hat{\theta}_{i}=\beta_{i} \int_{0}^{\infty} b(a)\left[\hat{\eta}_{i}(a)+\hat{\omega}_{i}(a)\right] d a
$$

for $i=1,2$. The local stability analysis of the general case has then been reduced to the study of the roots of equations (6.9) and (6.15). Unfortunately, the general case is complicated; however, the analysis is be carried out here for the special case when $\sigma_{1}=\sigma_{2}=0$. In this case recovery from one strain gives complete immunity to both strains so that no one ever enters classes $v_{1}, v_{2}$ and $w$. If

$$
H_{i}\left(\lambda^{*}\right)=\beta_{i} \int_{0}^{\infty} b(a) \rho e^{-M(a)} \int_{0}^{a} b(\alpha) e^{-\gamma_{i}(a-\alpha)-\lambda^{*} B(\alpha)} d \alpha d a,
$$

then the characteristic equations (6.9) for $\sigma_{1}=\sigma_{2}=0$ become $H_{1}\left(\lambda_{1}^{*}+\lambda_{2}^{*}\right)=1$ and $H_{2}\left(\lambda_{1}^{*}+\lambda_{2}^{*}\right)=1$ (note that each characteristic equation is similar to the characteristic equation (4.11) for the one-strain model), and the Lotka-type characteristic equation $(6.15)$ for $p$ reduces to

$$
\begin{aligned}
& \hat{\theta}_{i}=\beta_{i} \int_{0}^{\infty} b(a) \rho e^{-M(a)} \int_{0}^{a} b(\alpha) e^{-\gamma_{i}(a-\alpha)-\left(\lambda_{1}^{*}+\lambda_{i}^{*}\right) B(\alpha)} \\
& {\left[\hat{\theta}_{i} e^{-p(a-\alpha)}-\left(\hat{\theta}_{1}+\hat{\theta}_{2}\right) \lambda_{i}^{*} \int_{0}^{\alpha} b(\phi) e^{-p(a-\phi)} d \phi\right] d \alpha d a .}
\end{aligned}
$$

For the trivial steady-state age distribution $\left(\lambda_{1}^{*}=\lambda_{2}^{*}=0\right)$, equation (6.17) reduces to

$$
1=\beta_{i} \int_{0}^{\infty} b(a) p e^{-M(a)} \int_{0}^{a} b(\alpha) e^{-\left(p+\gamma_{i}\right)(a-\alpha)} d \alpha d a,
$$

which is similar to equation (5.16) for the one-strain model. Thus the analysis there can be used. If $H_{i}(0)<1$ for $i=1,2$, then the trivial steady-state age distribution is locally asymptotically stable. If $H_{i}(0)>1$ for $i=1,2$, then there is a positive real root of the characteristic equations so that the trivial steady-state age distribution is unstable.

A steady-state age distribution with $\lambda_{1}^{*}>0$ and $\lambda_{2}^{*}>0$ seems to occur only for very special parameter values, so we do not consider this case (see section 3). Consider the case when $H_{1}(0)>1$ and $H_{2}(0)<1$ so that there is a steady-state age distribution with $\lambda_{1}^{*}>0$ and $\lambda_{2}^{*}=0$. Now $H_{2}(0)<1$ implies that equation (6.18) with $i=2$ has only roots $p$ with negative real part so that this steady-state age distribution in which only the first strain persists is locally asymptotically stable. If $H_{2}(0)>1$, then there is a positive root $p$ so that this steady-state age distribution is unstable. There is also a steady-state age distribution in which only the second strain persists; this has analogous stability properties. Recall from 
Section 2 that the two-strain simulation model had periodic solutions for some age-specific mortalities, but not for exponential removal survivorship corresponding to a constant mortality rate $\mu$. Consequently, Andreasen (1988a, 1988b) considers the symmetric version of model (6.1)-(6.8) with $b(a)=1$ and age-specific mortality corresponding to a fixed lifespan of $A$ years for all individuals. We approximate the Lotka-type characteristic equation (6.15) by using the fact that the infectious period is four orders of magnitude smaller than the lifespan $A$ of the host. The approximate roots are purely imaginary, which suggests that there are periodic oscillations around the endemic steady state age distribution. Furthermore, the approximate period is $2 \pi \sqrt{K D / \sigma}$ where $D=1 / \gamma$ is the average infectious period, $K$ is the age at first infection, and $\sigma$ is the relative susceptibility due to cross-immunity. Thus the approximate results above and the simulation results in Section 2 are consistent.

\section{Discussion}

In his excellent survey paper Fine (1982) says: "Rather than continue to force influenza into simple epidemic theory an effort should be made to tackle some of the major puzzles of influenza patterns in large communities-the bimodal or undulating incidence pattern which is often observed, the apparent disappearance of virus for several months in large areas, or the recent recognition of widespread cocirculation of different shift viruses. Simulation techniques which incorporate important factors such as weather patterns, seasonal factors (e.g., school terms), or social geographic structure may be useful here." It is this challenge that motivates us to introduce and analyze (at different levels) models that incorporate age structure through age-dependent proportionately mixed contact rates, agedependent mortality rates, and interactions among viral strains or subtypes. It is the incorporation of two viral strains in our simulation model that produces the most interesting albeit the less reliable results. These two strains are coupled by a coefficient of cross-immunity $(\sigma)$; the coupling is strong when $\sigma$ is small (antigenically very similar strains) and weaker when $\sigma$ is intermediate (different strains same subtype). In both instances, the simulations yield sustained oscillations. Periods of 10 to 20 years are observed when $\sigma$ is small, while periods of 3-4 years are observed when cross-immunity is intermediate. These results are consistent with the recently documented evidence on the co-circulation of strains of the same subtype (Couch and Kasel, 1983; Thacker, 1986 [Fig. 2]). When only one strain is 
present, we have not found sustained oscillations; however, we observe very slowly damped oscillations. Hence, from a biological point of view, age structure by itself is capable of driving "sustained" (that is, slowly damped) oscillations. These results are in agreement with those previously reported by Anderson and May (1984) and Dietz and Schenzle (1985). When the age-structure is removed from the one- and two-strain models, we observe damped oscillations. Hence, for sustained oscillations, we require at least an age-structured population and two or more cocirculating viral strains. Age-structure enters this model through age-dependent activity levels and age-dependent mortality rates. Our simulations suggest that the interaction between cross-immunity and age-dependent survivorship may be enough to drive sustained oscillations, and that age-specific activity level rates with constant mortality are not sufficient (even in the presence of cross-immunity) to drive sustained oscillations. Variation of the transmission coefficients $\left(\beta_{1}, \beta_{2}\right)$ of our two viral strains seem to have significant effects on the amplitude of the oscillations. In contrast, the oscillations seem less sensitive to changes in the coefficient of cross-immunity $(\sigma)$ and slightly fluctuating transmission rates.

The consequences of co-circulating viruses within age-structured populations provide us with new insights and hypotheses into the dynamics of epidemics and pandemics. Our results suggest that the interactions between the immune system and multiple viruses could play a prominent role in the dynamics of viral infections such as influenza. The coefficient of cross-immunity provides a measure of this interaction between multiple strains of a virus. A very rough attempt at computing this parameter from the epidemiological data can be found in Castillo-Chavez, et al. (1988).

Further numerical and analytical investigations of the models presented would be helpful in achieving a more complete understanding of models related to influenza. In particular, it would be desirable to analyze further the steady state age distributions of the continuous age-structured model in Section 6 and to consider various models with time delays, and numerically integrate the systems of partial differential equations found in sections 4 and 6.

\section{Acknowledgments}

This research was conducted at the Cornell National Supercomputer Facility, Center for Theory and Simulation in Science and Engineering, which is funded, in part, by the National Science 
Foundation, New York State and IBM Corporation. We also acknowledge McIntire-Stennis grant (NYC-183568) and National Science Foundation grant DMS-8406472 to S. A. Levin. C. CastilloChavez acknowledges the support of the Office of the Provost and the Center for Applied Mathematics at Cornell University, as well as by a Ford Foundation Postdoctoral fellowship to minorities. W. Liu's work was partly supported by the U. S. Army Research Office through the Cornell University Mathematical Sciences Institute Fellowship. This manuscript has been improved considerably by helpful suggestions from the referees. 


\section{References}

Anderson, R.M. and May, R.M.: Spatial, temporal, and genetic heterogeneity in host populations and the design of immunization programmes. IMA J. of Math. Applied in Med. \& Biol. 1, 233-266 (1984).

Andreasen, V.: Dynamical models of epidemics in age-structured populations: Analysis and simplifications. Ph. D. thesis, Cornell University (1988a).

Andreasen, V.: Multiple time scales in the dynamics of infectious diseases. In (C. CastilloChavez, S. A. Levin, and C. Shoemaker, eds.) Mathematical Approaches to Ecological and Environmental Problem Solving. Lecture Notes in Biomathematics, Springer-Verlag, Heidelberg. (1988b, in preparation).

Barbour, A.D.: Macdonald's model and the transmission of bilharzia. Trans. Roy. Soc. Trop. Med. Hyg. 72, 6-15 (1978).

Beveridge, W.I.B.: Influenza: the last great plague. Heinemann, London (1977).

Busenberg, S., K. L. Cooke, and M. Iannelli. Stability and Thresholds in some AgeStructured Epidemics.In (C. Castillo-Chavez, S. A. Levin, and C. Shoemaker, eds.) Mathematical Approaches to Ecological and Environmental Problem Solving. Lecture Notes in Biomathematics, Springer-Verlag, Heidelberg. (1988, in preparation).

Castillo-Chavez, C., Hethcote, H.W., Andreasen, V., Levin, S.A., and Liu, W.: Crossimmunity in the dynamics of homogeneous and heterogeneous populations (1988). In Proc. of the Research Conference, Second Autumn Course in Mathematical Ecology, Trieste, 1986. World Scientific Publishing Co., Singapore (in press).

Couch, R.B. and Kasel, J.A.: Immunity to influenza in man. Ann. Rev. Microbial. 37, 529-549 (1983).

Dietz, K.: Transmission and control of arbovirus diseases. In Cooke, K.L. (ed.) Epidemiology, pp. 104-121. Society for Industrial and Applied Mathematics, Philadelphia (1975).

Dietz, K.: Epidemiologic interference of virus populations. J. Math. Biology. 8, 291-300 (1979).

Dietz, K. and Schenzle, D.: Proportionate mixing models for age-dependent infection transmission. J. Math. Biol. 22, 117-120 (1985).

Dowdle, W.R., Coleman, M.T., and Gregg, M.B.: Natural history of influenza type A in the United States; 1957-1972. Progr. Med. Viorl. 17, 91-135 (1974).

Elveback, L. R., and J. P. Fox, and A. Varma. An extension of the Reed-Frost Epidemic Model for the study of competition between viral agents in the presence of interference. Amer. J. Hyg. 80, 356-364 (1964).

Fine, P.: Applications of mathematical models to the epidemiology of influenza: a critique. In Selby, P. (ed.) Influenza Models, pp. 15-85. MTP Press, Lancaster, Boston, The Hague (1982). 
Fox, J.P., Kilbourne, E.D., and rapporteurs: From the National Institutes of Health: Epidemiology of influenza - summary of influenza workshop IV. J. Inf. Dis. 128, 361-386 (1973).

Hale, J.K.: Ordinary differential equations. Wiley-Interscience, New York (1969).

Hethcote, H.W.: Qualitative analysis for communicable disease models. Math. Biosci. 28, 335-356 (1976).

Hethcote, H.W., Stech, H.W., and van den Driessche, P.: Periodicity and stability in epidemic models: a survey. In Busenberg, S. and Cooke, K.L. (eds.) Differential equations and applications in ecology, epidemics and population problems, pp. 6582. Academic Press, New York (1981).

Hethcote, H.W. and Yorke, J.A.: Gonorrhea, transmission dynamics, and control. Springer, Berlin, Heidelberg, New York, Tokyo. Lecture Notes in Biomathematics 56 (1984).

Hethcote, H.W., and Van Ark, J.W.: Epidemiological models for heterogeneous populations: proportionate mixing, parameter estimation and immunization programs. Math. Biosci., to appear (1987).

Hoppensteadt, F.: An age dependent epidemic model. J. Franklin Inst. 297, 325-333 (1974).

Kilbourne, E.D.: The influenza viruses and influenza. Academic Press, New York (1975).

Longini, Jr. I.M., Ackerman, E., and Elveback, L.R.: An optimization model for influenza A epidemics. Math. Biosci. 38, 141-157 (1978).

MacKendrick, A.G.: Applications of mathematics to medical problems. Proc. Edinburgh Math. Soc. 44, 98-130 (1926).

May, R.M.: Population biology of microparasitic infections. In Hallam, T.G. and Levin, S.A. (eds.) Mathematical ecology, pp. 405-442. Springer, Berlin, Heidelberg (1986).

Nold, A.: Heterogeneity in diseases-transmission modeling. Math. Biosci. 52, 227-240 (1980).

Palese, P. and Young, F.J.: Variation of influenza A, B, and C viruses. Science 215, 1468-1474 (1982).

Ruelle, D. and Takens, F.: On the nature of turbulence. Communications in Mathematical Physics 20, 167-192 (1971).

Schaeffer, W.M. and Kot, M.: Nearly one dimensional dynamics in an epidemic. J. Theor. Biol. 112, 403-427 (1985).

Schenzle, D.: An age-structured model of pre- and post-vaccination measles transmission. IMA J. Math. Appl. Med. Biol. 1, 169-191 (1984). 
Schenzle, D: Control of virus transmission in age-structured populations. In Capasso, V., Grosso, E. and Paveri-Fontana, S.L. (eds.) Mathematics in Biology and Medicine, pp. 171-178. Lecture notes in Biomathematics 57. Springer, Berlin, Heidelberg (1985).

Selby, P. (ed.): Influenza: virus, vaccines, and strategy. (Proceedings of a working group on pandemic influenza, Rougemont, 26-28 January 1976). Academic Press, London and New York (1976).

Stuart-Harris, C.H. and Schild, G.C.: Influenza: the viruses and the disease. Edward Arnold, London (1976).

Thacker, S.B.: The persistence of influenza in human populations. Epidemiologic Reviews 8, 129-142 (1986).

Von Foerster, H.: Some remarks in changing populations. In Stohlman, Jr., F. (ed.) The kinetics of cellular proliferation, pp. 382-407. Frume and Stratton, New York (1959).

Webb, G.F.: Theory of nonlinear age-dependent population dynamics. Marcel Dekker, New York and Basel (1985).

Webster, R.G., Laver, W.G., Air, G.M., and Schild, G.C.: Molecular mechanisms of variation in influenza viruses. Nature 296, 115-121 (1982). 


\section{Appendix A}

Let $\lambda_{1}$ and $\lambda_{2}$ be the $G_{4}$ equilibrium values of $\beta_{1}\left(Y_{1}+V_{1}\right)$ and $\beta_{2}\left(Y_{2}+V_{2}\right)$, respectively. Since the infective replacement numbers (contact number times the susceptible fraction) $R_{1}\left(X+\sigma_{1} R_{2}\right)$ and $R_{2}$ $\left(X+\sigma_{2} R_{1}\right)$ are both one at a nontrivial equilibrium, then $\lambda_{1}$ and $\lambda_{2}$ satisfy the equations

$$
\begin{aligned}
& \left(\lambda_{1}+\lambda_{2}+\mu\right)\left(\sigma_{1} \lambda_{1}+\mu\right)=\mu R_{1}\left(\sigma_{1} \lambda_{1}+\sigma_{2} \lambda_{2} \gamma_{2} /\left(\gamma_{2}+\mu\right)+\mu\right), \\
& \left(\lambda_{1}+\lambda_{2}+\mu\right)\left(\sigma_{2} \lambda_{2}+\mu\right)=\mu R_{2}\left(\sigma_{2} \lambda_{2}+\sigma_{1} \lambda_{1} \gamma_{1} /\left(\gamma_{1}+\mu\right)+\mu\right) .
\end{aligned}
$$

The coordinates of the equilibrium $G_{4}$ are given by (3.14)-(3.17). In the symmetric case $\left(\beta_{1}=\beta_{2}=\beta\right.$, $\left.\gamma_{1}=\gamma_{2}=\gamma, \sigma_{1}=\sigma_{2}=\sigma\right)$ with symmetric initial conditions, the system (3.1)-(3.4) reduces to a four dimensional system

$$
\begin{gathered}
X^{\prime}(t)=-2 \beta(Y+V) X+\mu-\mu X \\
Y^{\prime}(t)=\beta(Y+V) X-(\gamma+\mu) Y \\
Z^{\prime}(t)=\gamma Y-(\sigma \beta(Y+V)+\mu) Z \\
V^{\prime}(t)=\sigma \beta(Y+V) Z-(\gamma+\mu) V
\end{gathered}
$$

where $\mathrm{W}(\mathrm{t})$ can be found from $X+2 Y+2 Z+2 V+W=1$. The existence of a nontrivial equilibrium corresponding to the equilibrium $G_{4}$ is guaranteed provided that the threshold condition $R=\beta /(\gamma+\mu)>1$ is satisfied. The Jacobian of the system (A3)-(A6) is

$$
J=\left(\begin{array}{cccc}
-(2 \lambda+\mu) & 0 & -2 \beta X & -2 \beta X \\
0 & -(\sigma \lambda+\mu) & -\sigma \beta Z & -\sigma \beta Z+\gamma \\
0 & \sigma \lambda & \sigma \beta Z-\gamma-\mu & \sigma \beta Z \\
\lambda & 0 & \beta X & \beta X-\gamma-\mu
\end{array}\right)
$$

At the nontrivial equilibrium, the equations (A1) and (A2) reduce to

$$
(2 \lambda+\mu)(\sigma \lambda+\mu)=\mu \beta[\sigma \lambda(2 \gamma+\mu)+\mu(\gamma+\mu)] /(\gamma+\mu)^{2}
$$

Using the expressions (3.14) and (3.16) for $X$ and $Z$ and equation (A8) for $\beta$, the characteristic equation $\operatorname{det}(J-s I)=0$ for the nontrivial equilibrium reduces to the following equation in the four parameters $\gamma$, $\mu, \sigma$ and $\lambda$.

$$
\begin{gathered}
{\left[\gamma(2 \sigma \lambda+\mu)+(\mu(\sigma \lambda+\mu)](2 \lambda+\mu+s)(\sigma \lambda+\mu+s)(\gamma+\mu+s)^{2}\right.} \\
-(\gamma+\mu)^{2}(\sigma \lambda+\mu)(\mu+s)[(\sigma \lambda+\mu+s)(\gamma+\mu+s)+\sigma \lambda \gamma] \\
-\sigma \lambda \gamma(\gamma+\mu)(2 \lambda+\mu+s)(\gamma+\mu+s)(\mu+s)=0
\end{gathered}
$$

We have shown using the symbolic manipulator MACSYMA that the Routh-Hurwitz criteria are satisfied for the fourth degree polynomial equation (A9) so that all eigenvalues have negative real parts. 
Thus this nontrivial equilibrium is locally asymptotically stable. 


\section{Appendix B}

The steady-state age-distributions and characteristic equations are now described for the continuous two-strain model (6.1)-(6.8). Assume that steady-state age-distributions are approached as time approaches infinity so that the forces of infection are asymptotically constants denoted by $\lambda_{1}^{*}$ and $\lambda_{2}^{*}$. The following steady-state age distributions with $B(a)=\int_{0}^{a} b(\alpha) d \alpha$ and $M(a)=\int_{0}^{a} \mu(\alpha) d \alpha$ are solutions of (5.1)-(5.10) that are independent of $t$.

$$
\begin{gathered}
x^{*}(a)=\rho e^{-M(a)} \exp \left[-\left(\lambda_{1}^{*}+\lambda_{2}^{*}\right) B(a)\right], \\
y_{i}^{*}(a)=\rho e^{-M(a)} \int_{0}^{a} \lambda_{i}^{*} b(\alpha) e^{-\gamma_{i}(a-\alpha)-\left(\lambda_{1}^{*}+\lambda_{2}^{*}\right) B(\alpha)} d \alpha, \\
z_{i}^{*}(a)=\rho e^{-M(a)} \int_{0}^{a} \gamma_{i} \lambda_{i}^{*} e^{-\sigma_{j} \lambda_{j}^{*}[B(a)-B(\alpha)]}\left[\int_{0}^{\alpha} b(\phi) e^{-\gamma_{i}(\alpha-\phi)-\left(\lambda_{1}^{*}+\lambda_{2}^{*}\right) B(\phi)} d \phi\right] d \alpha, \\
v_{i}^{*}(a)=\rho e^{-M(a)} \int_{0}^{a} \sigma_{i} \lambda_{i}^{*} b(\alpha) e^{-\gamma_{i}(a-\alpha)}\left[\int_{0}^{\alpha} \gamma_{j} \lambda_{j}^{*} e^{-\sigma_{i} \lambda_{i}^{*}[B(\alpha)-B(\theta)]}\right. \\
\left.\left.\int_{0}^{\theta} b(\phi) e^{-\gamma_{j}(\theta-\phi)-\left(\lambda_{1}^{*}+\lambda_{i}^{*}\right) B(\phi)} d \phi\right) d \theta\right] d \alpha, \\
w^{*}(a)=\rho e^{-M(a)}-x^{*}(a)-y_{1}^{*}(a)-y_{2}^{*}(a)-v_{1}^{*}(a)-v_{2}^{*}(a)-z_{1}^{*}(a)-z_{2}^{*}(a)
\end{gathered}
$$

Substituting $y_{i}^{*}(a)$ and $v_{i}^{*}(a)$ in

$$
\lambda_{i}^{*}=\beta_{i} \int_{0}^{\infty} b(\alpha)\left[y_{i}^{*}(\alpha)+v_{i}^{*}(\alpha)\right] d \alpha
$$

we find that either $\lambda_{i}^{*}=0$ or $\lambda_{i}^{*}$ satisfies the characteristic equations

$$
\begin{gathered}
1=\beta_{i} \int_{0}^{\infty} b(a) p e^{-M(a)}\left[\int _ { 0 } ^ { a } b ( \alpha ) e ^ { - \gamma _ { i } ( a - \alpha ) } \left(e^{-\left(\lambda_{1}^{*}+\lambda_{2}^{*}\right) B(\alpha)}\right.\right. \\
\left.\left.+\sigma_{i} \gamma_{j} \lambda_{j}^{*} \int_{0}^{\alpha} e^{-\sigma_{i} \lambda_{i}^{*}(B(\alpha)-B(\theta))}\left[\int_{0}^{\theta} b(\phi) e^{-\gamma_{j}(\theta-\phi)-\left(\lambda_{1}^{*}+\lambda_{2}^{*}\right) B(\phi)} d \phi\right] d \theta\right) d \alpha\right] d a,
\end{gathered}
$$

$(i, j)=(1,2)$ and $(i, j)=(2,1)$. 


\section{Appendix C}

Let us consider the following perturbation solutions from the steady-state age distributions to the system (6.1)-(6.8)

$$
\begin{gathered}
x(a, t)=x^{*}(a)+\xi(a, t), \\
y_{i}(a, t)=y_{i}^{*}(a)+\eta_{i}(a, t), \\
z_{i}(a, t)=z_{i}^{*}(a)+\rho_{i}(a, t), \\
v_{i}(a, t)=v_{i}^{*}(a)+\omega_{i}(a, t), \\
\lambda_{i}(t)=\lambda_{i}^{*}+\theta_{i}(t),
\end{gathered}
$$

where $0=\xi(0, t)=\eta_{i}(0, t)=\rho_{i}(0, t)=\omega_{i}(0, t)$ for $i=1,2$. Neglecting terms of order higher than one, we find that $\xi, \eta_{i}, \rho_{i}, \omega_{i}$ and $\hat{\theta}_{i}$ satisfy the following linear system

$$
\begin{gathered}
\left(\frac{\partial}{\partial a}+\frac{\partial}{\partial t}\right) \xi(a, t)=-\left(\lambda_{1}^{*}+\lambda_{2}^{*}\right) b(a) \xi(a, t)-b(a)\left[\theta_{1}(t)+\theta_{2}(t)\right] x^{*}(a)-\mu(a) \xi(a, t), \\
\left(\frac{\partial}{\partial a}+\frac{\partial}{\partial t}\right) \eta_{i}(a, t)=\lambda_{i}^{*} b(a) \xi(a, t)-\left(\mu(a)+\gamma_{i}\right) \eta_{i}(a, t)+b(a) \theta_{i}(t) x^{*}(a), \\
\left(\frac{\partial}{\partial a}+\frac{\partial}{\partial t}\right) \rho_{i}(a, t)=-\sigma_{j} b(a) \lambda_{j}^{*} \rho_{i}(a, t)-\sigma_{j} b(a) \theta_{j}(t) z_{i}^{*}(a)+\gamma_{i} \eta_{i}(a, t)-\mu(a) \rho_{i}(a, t), \\
\left(\frac{\partial}{\partial a}+\frac{\partial}{\partial t}\right) \omega_{i}(a, t)=\sigma_{i} b(a) \lambda_{i}^{*} \rho_{j}(a, t)+\sigma_{i} b(a) \theta_{i}(t) z_{j}^{*}(a)-\left[\gamma_{i}+\mu(a)\right] \omega_{i}(a, t), \\
\theta_{i}(t)=\beta_{i} \int_{0}^{\infty} b(a)\left[\eta_{i}(a, t)+\omega_{i}(a, t)\right] d a .
\end{gathered}
$$

Separable perturbations of the form $A(a) T(t)$ require that $T(t)=e^{p t}$. Hence we consider perturbations of the form $\xi(a) e^{p t}, \hat{\eta}_{i}(a) e^{p t}, \hat{\rho}_{i}(a) e^{p t}, \hat{\omega}_{i}(a) e^{p t}$, and $\hat{\theta}(a) e^{p t}$, where $\hat{\theta}$ is a constant. We then find that:

$$
\begin{gathered}
\xi(a)=-\rho e^{-M(a)-\left(\lambda_{1}^{*}+\lambda_{2}^{*}\right) B(a)}\left(\hat{\theta}_{1}+\hat{\theta}_{2}\right) \int_{0}^{a} b(\alpha) e^{-p(a-\alpha)} d \alpha, \\
\eta_{i}(a)=\rho e^{-M(a)} \int_{0}^{a} b(\alpha) e^{-\gamma_{i}(a-\alpha)-\left(\lambda_{1}^{*}+\lambda_{2}^{*}\right) B(\alpha)}\left[\hat{\theta}_{i} e^{-p(a-\alpha)}-\left(\hat{\theta}_{1}+\hat{\theta}_{2}\right) \lambda_{i}^{*} \int_{0}^{\alpha} b(\phi) e^{-p(a-\phi)} d \phi\right] d \alpha .
\end{gathered}
$$

The expressions for $\hat{p}_{i}(a)$ and $\hat{\omega}_{i}(a)$ are omitted since they are very complicated. When $\sigma_{1}=\sigma_{2}=0$, then no one enters the classes $v_{1}, v_{2}$, and $w$. Hence equation (C10) reduces to

$$
\hat{\theta}_{i}=\int_{0}^{\infty} b(a) \hat{\eta}_{i}(a) d a
$$

After substituting (C12) into (C13), we arrive at the following Lotka-type characteristic equation for $p$

$$
\hat{\theta}_{i}=\beta_{i} \int_{0}^{\infty} b(a) \rho e^{-M(a)} \int_{0}^{a} b(\alpha) e^{-\gamma_{i}(a-\alpha)-\left(\lambda_{1}^{*}+\lambda_{2}^{*}\right) B(\alpha)}
$$


$\left[\hat{\theta}_{i} e^{-p(a-\alpha)}-\left(\hat{\theta}_{1}+\hat{\theta}_{2}\right) \lambda_{i}^{*} \int_{0}^{\alpha} b(\phi) e^{-p(a-\phi)} d \phi\right] d \alpha d a$. 


\section{Appendix D}

In this appendix we show that the models (4.1)-(4.6) and (6.1)-(6.8) are well posed. The method of proof is similar to that used by Hoppensteadt (1974) to show the well-posedness of a very general one agent age dependent epidemic model. First let $U(a, t)=\operatorname{col}\left[x, y_{1}, z_{1}, y_{2}, z_{2}, v_{1}, v_{2}, w\right]$ and let $A$ denote the matrix

$$
\left[\begin{array}{cccccccc}
\left(\lambda_{1}+\lambda_{2}\right) b(a)+\mu(a) & 0 & 0 & 0 & 0 & 0 & 0 & 0 \\
-\lambda_{1}(t) b(a) & \gamma_{1}+\mu(a) & 0 & 0 & 0 & 0 & 0 & 0 \\
0 & -\gamma_{1} & \sigma_{2} \lambda_{2}(t) b(a)+\mu(a) & 0 & 0 & 0 & 0 & 0 \\
-\lambda_{2}(t) b(a) & 0 & 0 & \gamma_{2}+\mu(a) & 0 & 0 & 0 & 0 \\
0 & 0 & 0 & -\gamma_{2} & \sigma_{1} \lambda_{1}(t) b(a)+\mu(a) & 0 & 0 & 0 \\
0 & 0 & 0 & 0 & -\sigma_{1} \lambda_{1}(t) b(a) & \gamma_{1}+\mu(a) & 0 & 0 \\
0 & 0 & -\sigma_{2} \lambda_{2}(t) b(a) & 0 & 0 & 0 & \gamma_{2}+\mu(a) & 0 \\
0 & 0 & 0 & 0 & 0 & -\gamma_{1} & -\gamma_{2} & \mu(a)
\end{array}\right] .
$$

Then the two-strain model is given by

$$
\left(\frac{\partial}{\partial t}+\frac{\partial}{\partial a}\right) U+A(U) U=0,
$$

which along characteristics parametrized by $s$ is given by the system

$$
\frac{d}{d s} U=-A(U) U ; U(0)=\left\{\begin{array}{l}
U(0, t)=\operatorname{col}[p, 0,0,0,0,0,0,0], t>0, \\
U(a, 0)=\operatorname{col}\left[x_{0}(a), y_{01}(a), z_{1}(a), y_{02}, z_{2}, v_{01}, v_{02}, z_{0}\right], a>0 .
\end{array}\right.
$$

Since $-A(U)$ has only non-negative off-diagonal terms, all solutions to (6.15) are nonnegative for $s \geq 0$ provided that $U \geq 0$ at $s=0$. If we now let

$$
n(a, t)=\|U(a, t)\|=x(a, t)+y_{1}(a, t)+y_{2}(a, t)+z_{1}(a, t)+z_{2}(a, t)+v_{1}(a, t)+v_{2}(a, t)+w(a, t),
$$

then the dynamics of $n(a, t)$ obeys a particular case of the MacKendrick (1926) / Von Foerster (1957) model, namely:

$$
\begin{gathered}
\left(\frac{\partial}{\partial a}+\frac{\partial}{\partial t}+\mu(a)\right) n(a, t)=0 \\
n(0, t)=\rho \\
n(a, 0)=\|U(a, 0)\| .
\end{gathered}
$$

Our formulation (6.1)-(6.8) is chosen so that the total population probability density $n(a, t)$ is at the steady-state age distribution given by $\rho{ }^{4(a)}$ before the disease dynamics starts. Thus the epidemiologic dynamics are not complicated by simultaneous demographic dynamics. Our choice of 
$\rho=\left[\int_{0}^{\infty} e^{-M\left(a^{\prime}\right)} d a^{\prime}\right]^{-1}$ implies that the total population size has been normalized to 1 . With the a priori bound given by 1 , the existence and uniqueness proof is now exactly the same as the well known contraction mapping proof for initial value problems of systems of ordinary differential equations. Moreover, the a priori bound shows that the solution can be continued for all time. Continuous dependence on the data follows from known results on continuous dependence of fixed points to parameterdependent contraction maps. 
Table 1

\begin{tabular}{|c|c|c|c|c|}
\hline $\mathrm{A}$ & $\beta$ & $\mathrm{I}$ & $\mathrm{a}$ & $\mathrm{b}$ \\
\hline $1: 4: 4: 4: 4$ & 0.10 & $7.2 \times 10^{-5}$ & $-3.1 \times 10^{-4}$ & 0.11 \\
$1: 1: 1: 1: 1$ & 2.95 & $1.3 \times 10^{-4}$ & $-2.7 \times 10^{-4}$ & 0.19 \\
$1: 8: 1: 1: 1$ & 0.23 & $1.2 \times 10^{-4}$ & $-2.8 \times 10^{-3}$ & 0.35 \\
$1: 4: 4: 4: 8$ & 0.195 & $1.3 \times 10^{-4}$ & $-3.0 \times 10^{-4}$ & 0.15 \\
$4: 8: 4: 2: 1$ & 0.10 & $7.4 \times 10^{-5}$ & $-1.9 \times 10^{-3}$ & 0.22 \\
$4: 8: 4: 2: 1$ & 0.13 & $1.1 \times 10^{-4}$ & $-9.0 \times 10^{-4}$ & 0.25 \\
\hline $4: 8: 2: 4: 1$ & 0.15 & $1.2 \times 10^{-4}$ & $-9.0 \times 10^{-4}$ & \\
\hline
\end{tabular}

Dominant eigenvalues for the non-trivial equilibrium in the one-strain age-structure model with proportionate mixing for different sets of activity levels. The first column (A) shows the activity levels for the different age categories; the second gives the incidence proportionality factors $(\beta)$, the third gives the equilibrium proportion of infected individuals (I), and the fourth and fifth give the real and imaginary part of the dominant eigenvalues. For more details see the text. 
Table 2

\begin{tabular}{|l|l|l|l|l|l|}
\hline$\sigma_{1}$ & $\sigma_{2}$ & $\operatorname{Re}\left(\delta_{1}\right)$ & $\operatorname{Im}\left(\delta_{1}\right)$ & $\operatorname{Re}\left(\delta_{2}\right)$ & $\operatorname{Im}\left(\delta_{2}\right)$ \\
\hline 0.9 & 0.9 & $-3.767 \times 10^{-5}$ & $4.136 \times 10^{-3}$ & $-6.514 \times 10^{-5}$ & $4.683 \times 10^{-3}$ \\
0.9 & 0.8 & $-3.137 \times 10^{-5}$ & $4.076 \times 10^{-3}$ & $-7.137 \times 10^{-5}$ & $4.603 \times 10^{-3}$ \\
0.9 & 0.4 & $-2.790 \times 10^{-5}$ & $3.581 \times 10^{-3}$ & $-7.696 \times 10^{-5}$ & $4.423 \times 10^{-3}$ \\
\hline 0.8 & 0.9 & $-3.655 \times 10^{-5}$ & $3.946 \times 10^{-3}$ & $-6.622 \times 10^{-5}$ & $4.704 \times 10^{-3}$ \\
0.8 & 0.8 & $-3.184 \times 10^{-5}$ & $3.869 \times 10^{-3}$ & $-7.031 \times 10^{-5}$ & $4.643 \times 10^{-3}$ \\
0.8 & 0.4 & $-2.670 \times 10^{-5}$ & $3.369 \times 10^{-3}$ & $-7.546 \times 10^{-5}$ & $4.477 \times 10^{-3}$ \\
\hline 0.4 & 0.9 & $-3.964 \times 10^{-5}$ & $3.024 \times 10^{-3}$ & $-6.571 \times 10^{-5}$ & $4.746 \times 10^{-3}$ \\
0.4 & 0.8 & $-3.533 \times 10^{-5}$ & $2.927 \times 10^{-3}$ & $-6.715 \times 10^{-5}$ & $4.719 \times 10^{-3}$ \\
\hline 0.4 & $-2.300 \times 10^{-5}$ & $2.453 \times 10^{-3}$ & $-6.959 \times 10^{-5}$ & 0 \\
\hline
\end{tabular}

Dominant and subdominant eigenvalues, $\delta_{1}$ and $\delta_{2}$, for the nontrivial equilibrium in the two-strain model for different values of $\sigma_{1}$ and $\sigma_{2}\left(\beta_{1}=0.8, \beta_{2}=0.9, \gamma_{1}=\gamma_{2}=0.33, \mu=\right.$ 0.00004). All the eigenvalues have negative real parts including those not shown here. The imaginary part of the dominant eigenvalue is about 100 times larger than its real part. 


\section{Figure Legends}

Fig. 1. Transfer diagram for a single viral strain on a single host population. The variables $X, Y$, and $Z$ are defined in the text.

Fig. 2. Transfer diagram for two co-circulating viral strains or subtypes on a single host population. The variables $X, Y_{i}, Z_{i}, V_{i}$ and $W$ are defined in the text.

Fig. 3. Proportion of the population infected with strain $1, I_{1}$ when $\sigma=0.05$; i.e., very strong crossimmunity. The incidences of the two strains are strongly coupled and the system will go through cycles wi: period of 20 years, $\beta=0.20$ and the infectious period (=time step) is 3 days.

Fig. 4. Proportion of the population infected with strain $1, I$, when $\sigma=0.34$ (smaller amplitude), $\sigma=0.4$ (intermediate amplitude), and $\sigma=0.5$ (larger amplitude). For these intermediate values of $\sigma, I_{1}$ cycles with a period of $3-4$ years $(\beta=0.20$ and infectious period is 3 days).

Fig. 5. For $\sigma=0.32$ and $\beta=0.20$, the model exhibits very complicated dynamics, switching between two unstable spirals. The trajectories are shown here in the coordinate system $\left(I_{1}(t), I_{1}\left(t+T_{1}\right), I_{1}\left(t+T_{2}\right)\right)$, $T_{1}=300$ days, $T_{2}=600$ days. Note that $T_{1}$ is about half the period of the oscillation and $T_{2}=2 T_{1}$. Coordinate systems of this kind were proposed by Ruelle and Takens (1971) and first used in ecological models by Schaffer and Kot (1985). Technically and practically, Ruelle-Takens' coordinate systems may provide more insightful dynamical representations.

Fig. 6 a. Sensitivity of the regular cycles $\left(\sigma_{1}=\sigma_{2}=0.50, \beta_{1}=0.15\right)$ to changes in $\beta_{2}, \beta_{2}=0.15,0.17,0.18$. The amplitude of the cycle decreases with increasing $\beta_{2}$. When the transmission coefficients, and thereby the time scales of the two strains, become different, the disease dynamics decouples.

Fig. 6 b. Proportion of the population infected with strain $1, I_{1}$, when $\beta_{1}=.15$ and $\beta_{2}=.15$ (larger amplitude), $\beta_{2}=.17$ (intermediate amplitude), and $\beta_{3}=.18$ (smaller amplitude). The coordinate system (not shown for clarity) is as in Fig. 4. 
Fig. 7. Sensitivity of the regular cycles $\left(\sigma_{1}=0.50, \sigma_{2}=0.50, \beta_{1}=\beta_{2}=0.20\right)$ to changes in the crossimmunity of the strain $\sigma_{2}$, where $\sigma_{2}=0.50,0.55,0.60$. The coordinate system is as in Fig. 4 . The cycles change very little when $\sigma_{2}$ changes.

Fig. 8. The effect of a small seasonal variation in the transmission coefficient $\beta=0.20+0.005 \sin \omega t$ where $\omega=2 \pi /$ year, $\sigma=0.50$. The coordinate system is as in Fig. 4 . 
$\mathrm{S} \longrightarrow \mathrm{I} \longrightarrow \mathrm{R}$.

Figure 1 


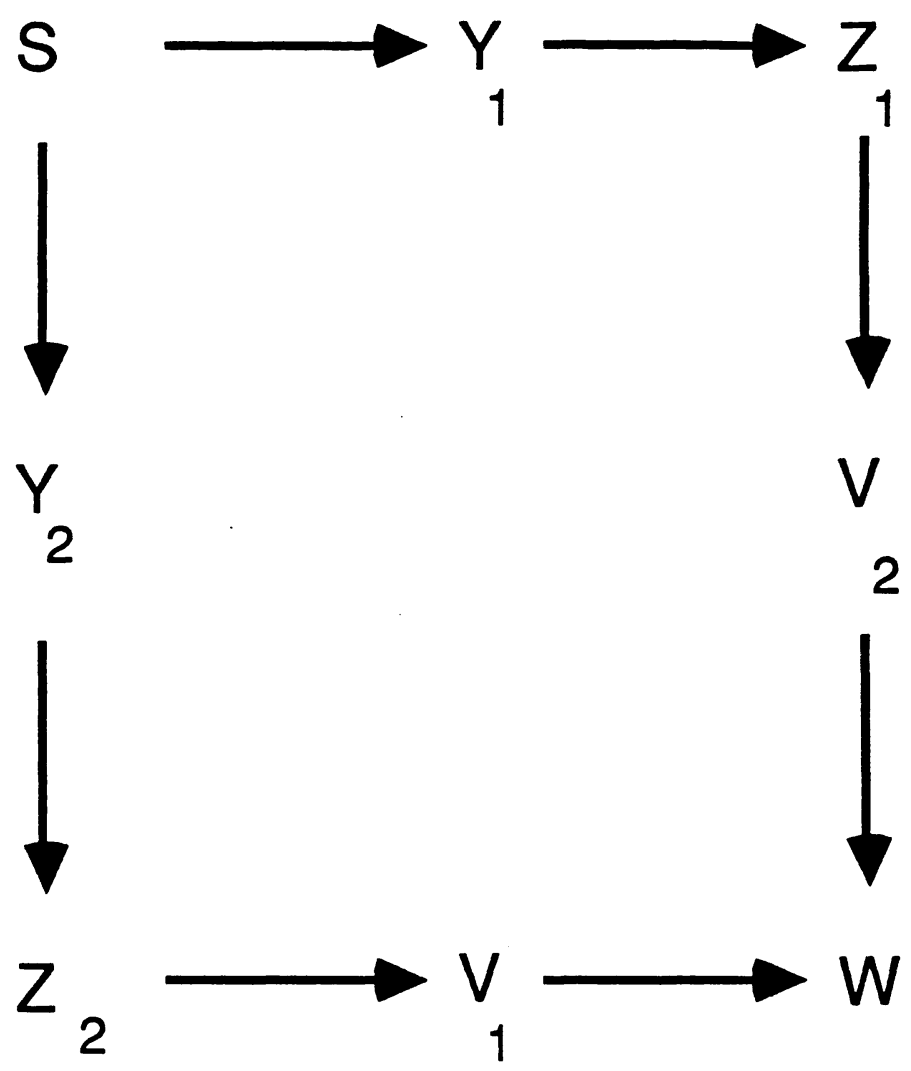

Figure 2 


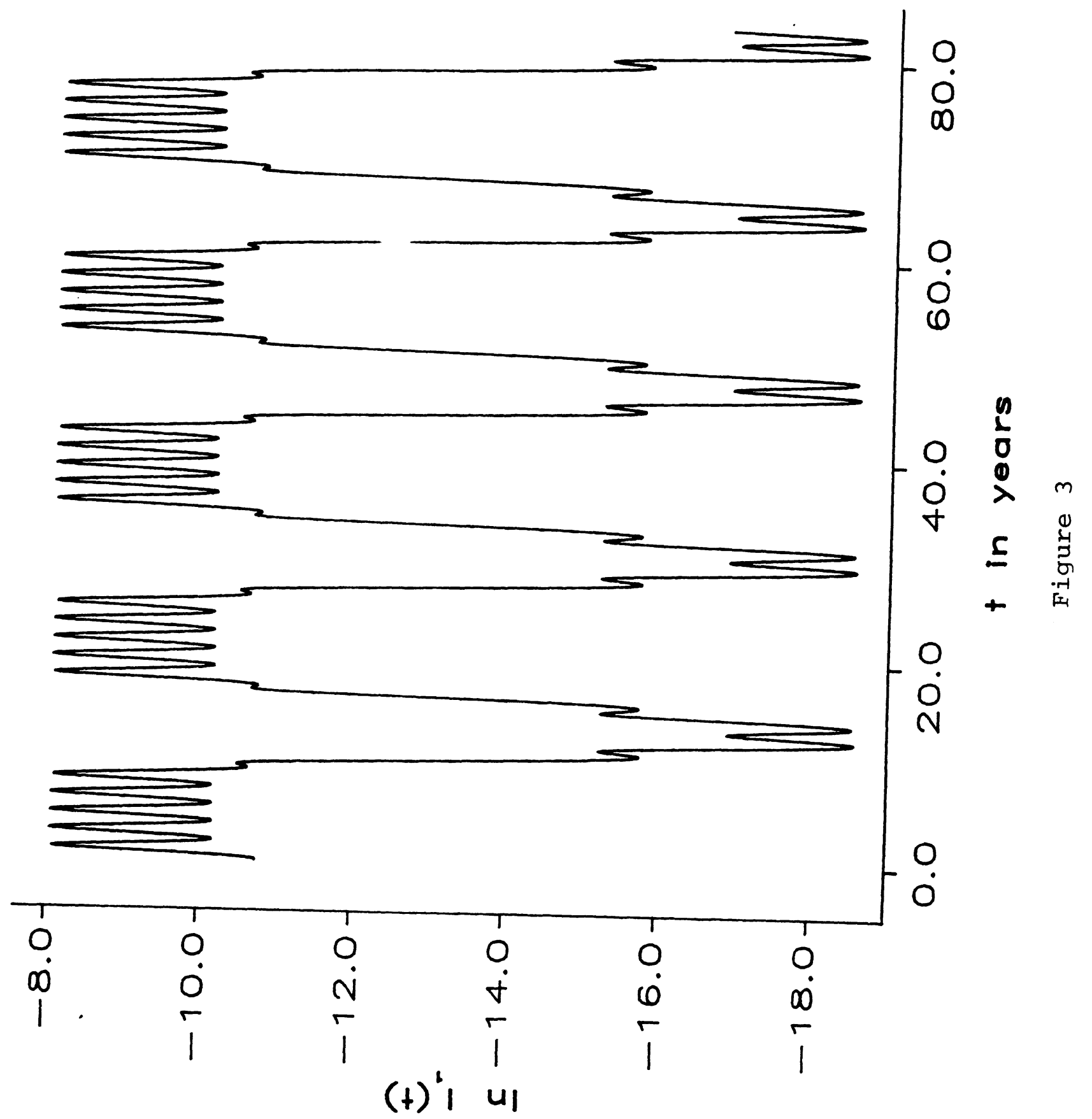




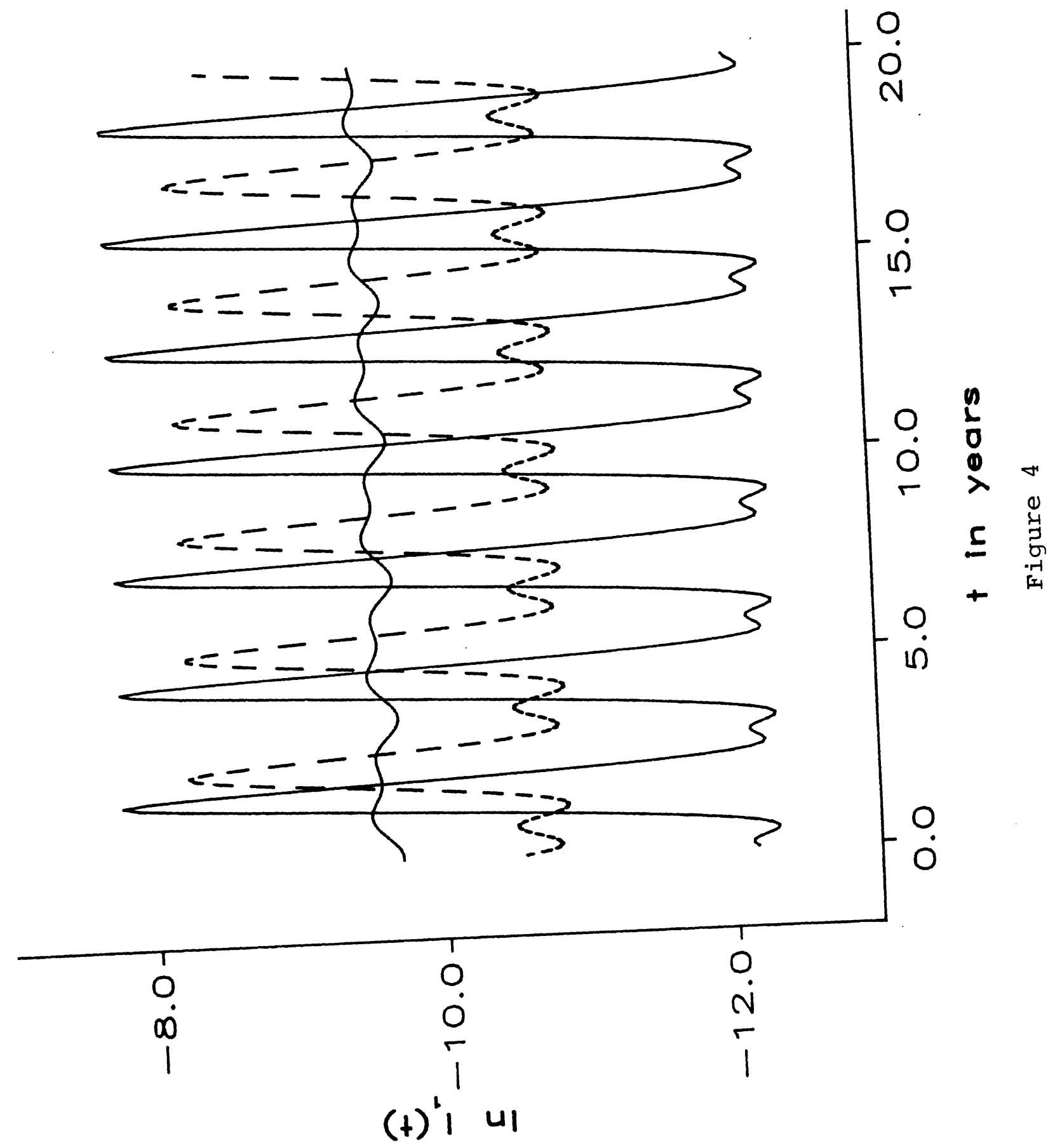




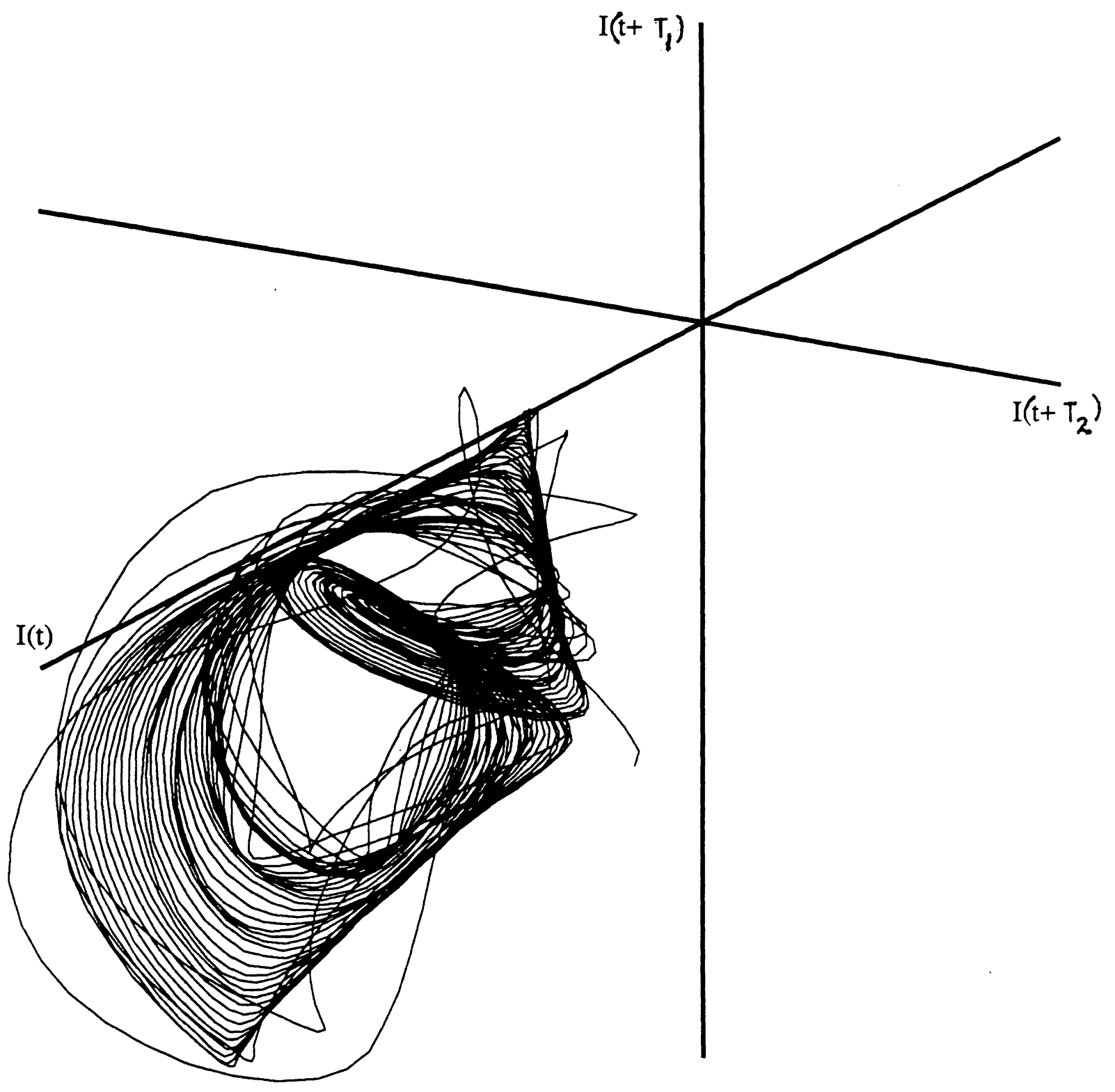

Figure 5 


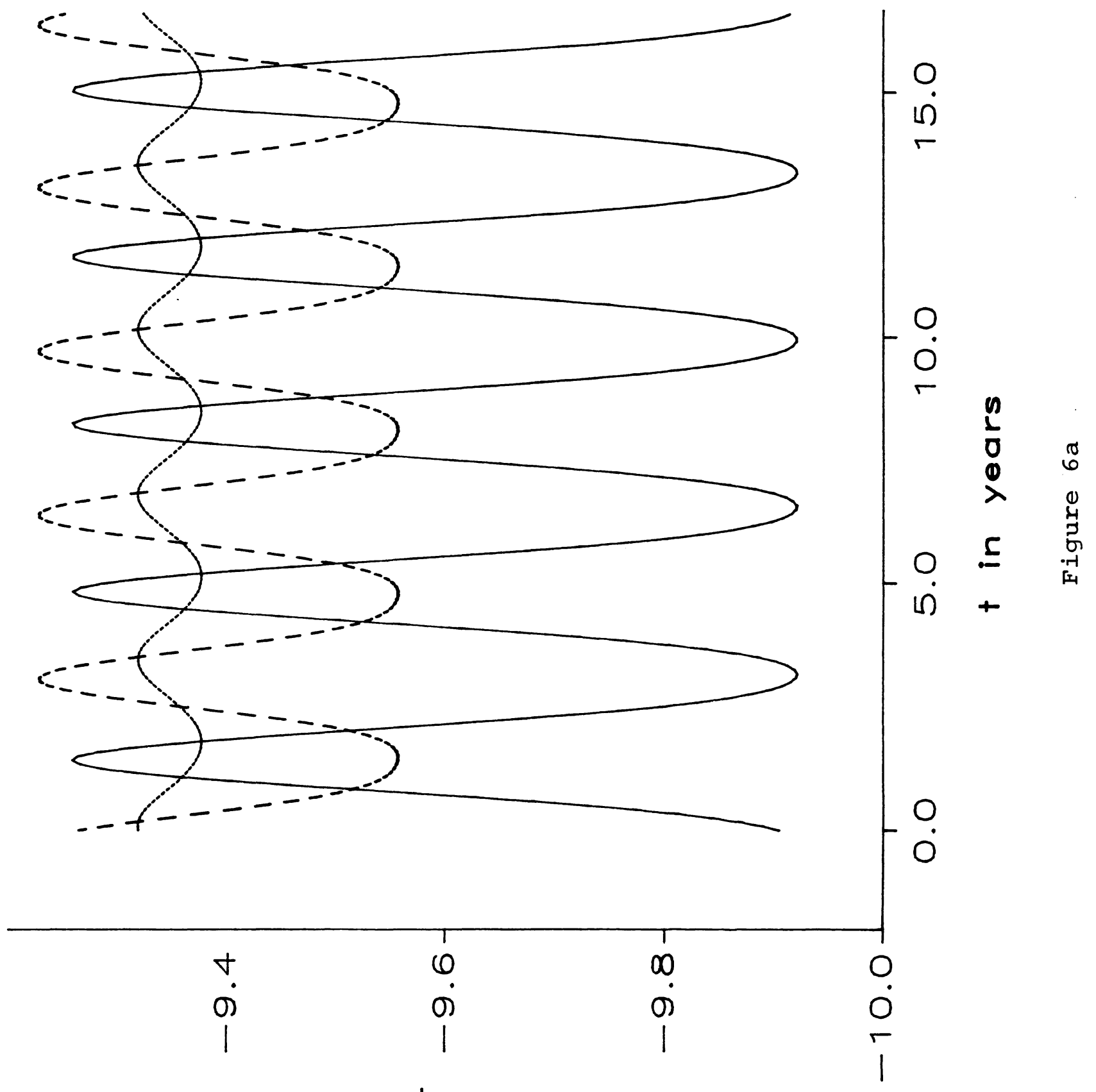

(t) । 


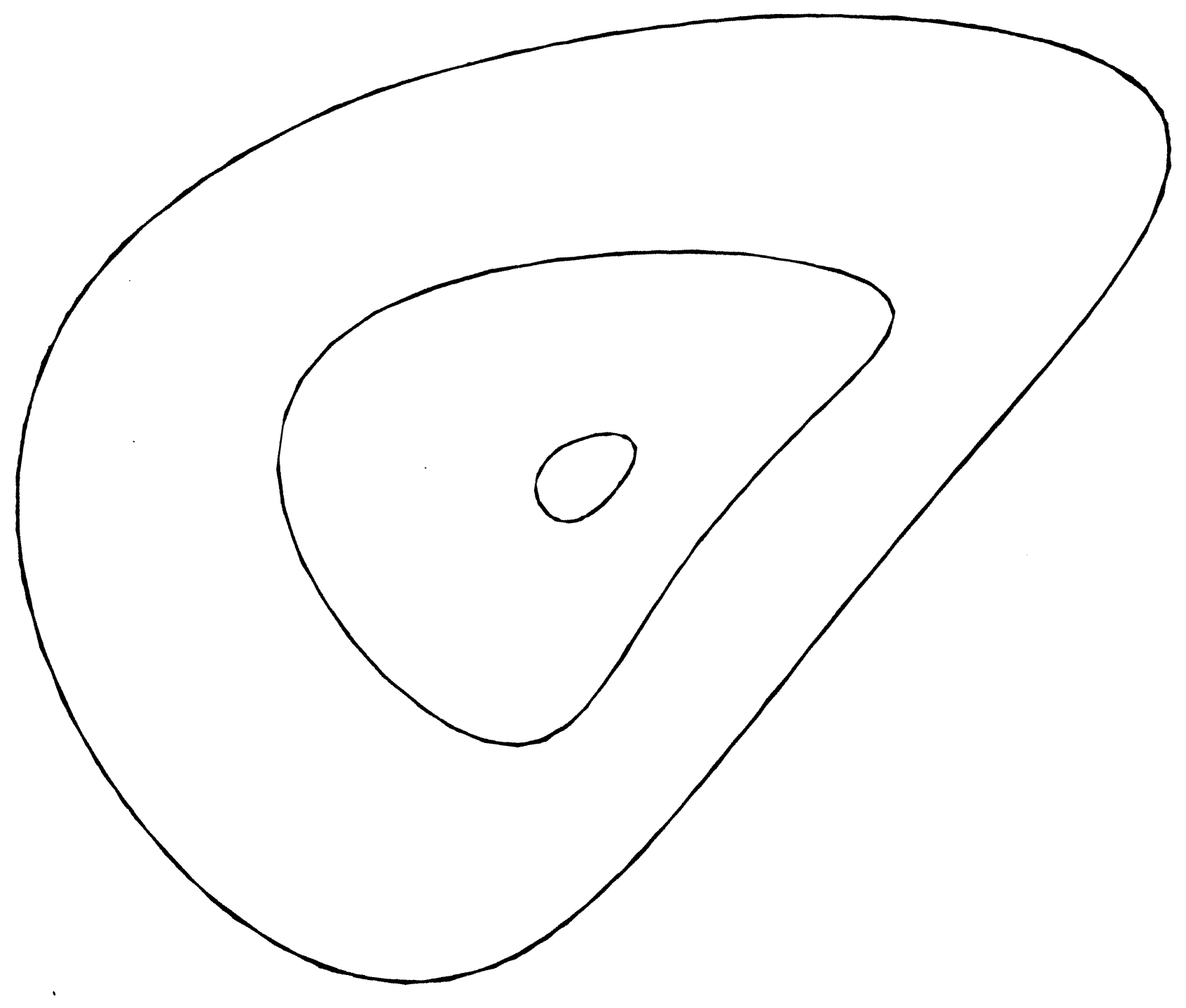

Figure $6 \mathrm{~b}$ 


$$
\Delta
$$




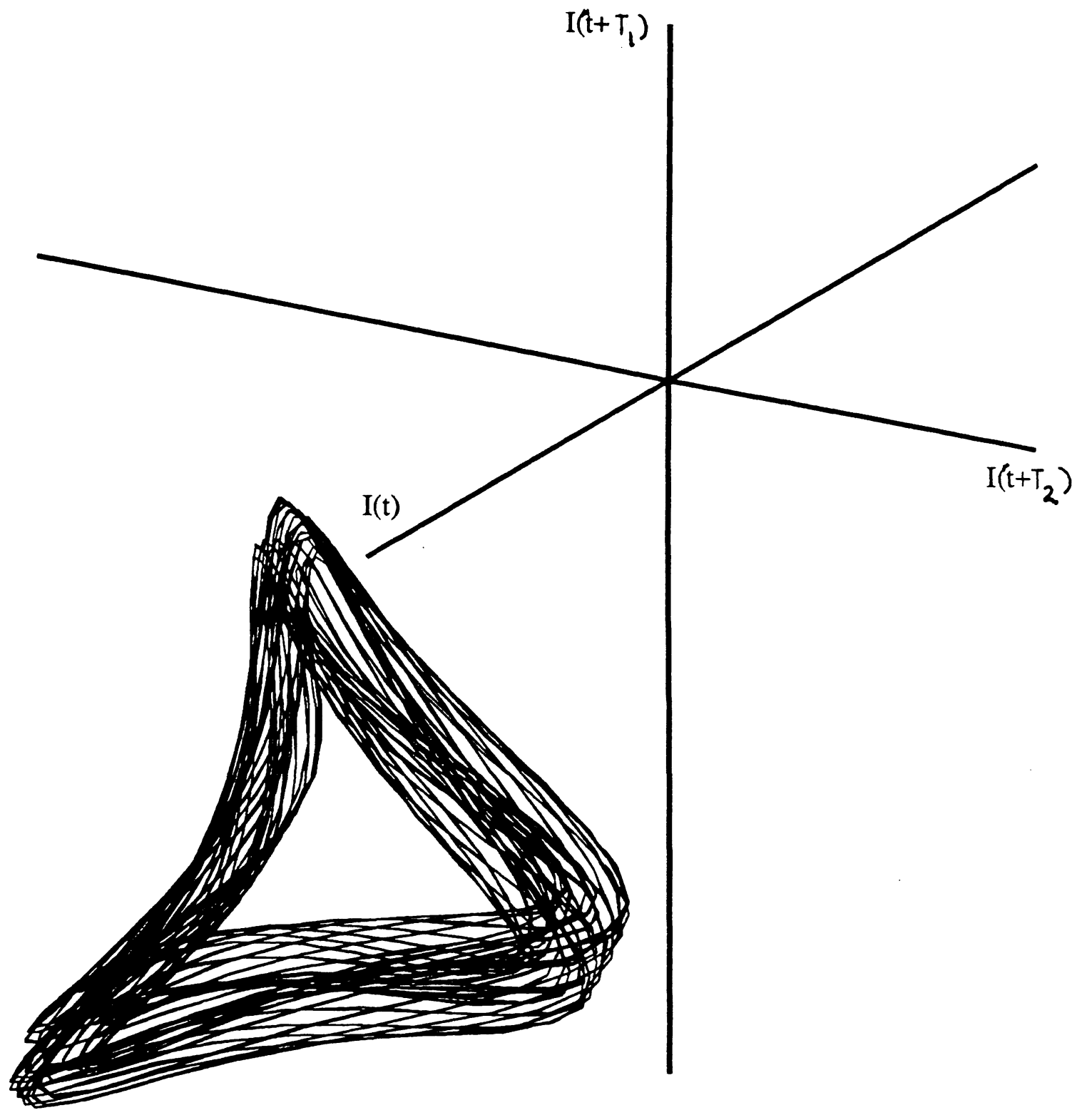

Figure 8 